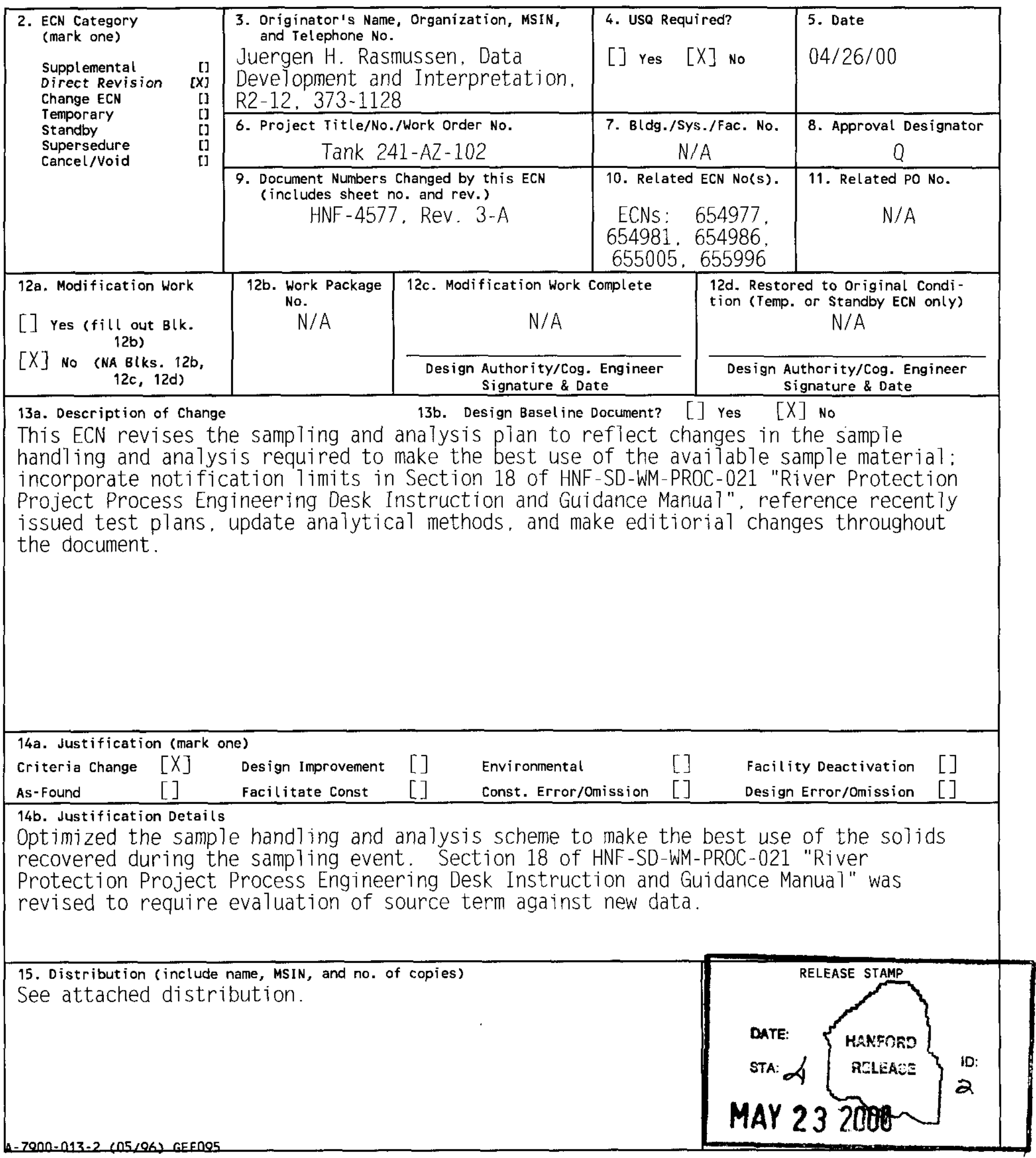




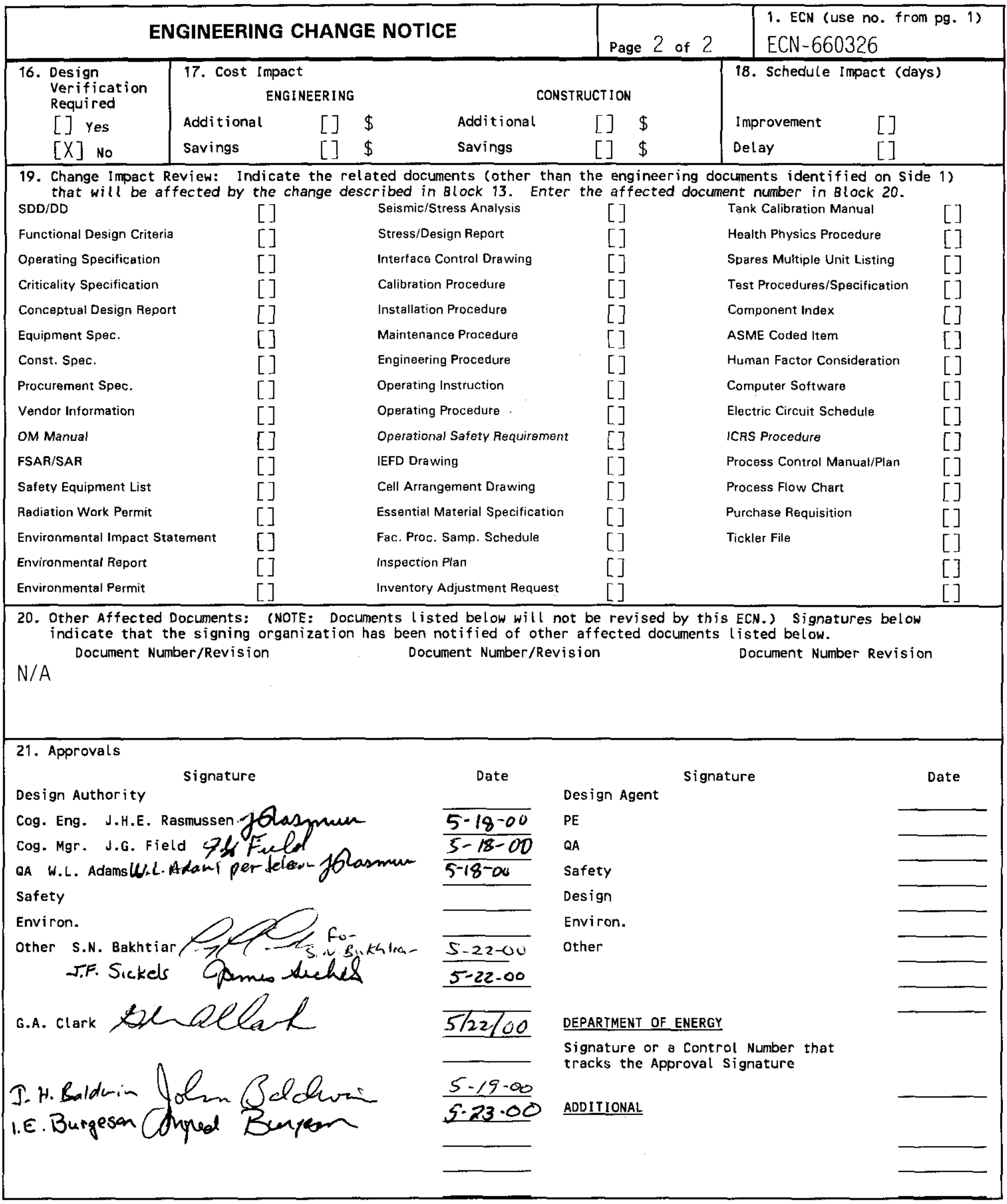




\section{Tank 241-AZ-102 Privatization Push Mode Core Sampling and Analysis Plan}

Juergen $H$. Rasmussen

CH2M HILL Hanford Gruop. Inc., Richland. WA 99352

U.S. Department of Energy Contract DE-AC06-96RL13200

EDT/ECN: ECN-660326

Org Code: $74 \mathrm{~B} 20$

UC: 2070

CACN/COA: 102264/ES11

B\&R Code: EW 3120074

Total Pages: 48

Key Words: Tank 241-AZ-102, 241-AZ-102, Tank AZ-102, AZ-102, AZ Farm, Privatization. Push Mode. Push, Core, Sampling. Analysis

Abstract: $N / A$

TRADEMARK DISCLAIMER. Reference herein to any specific commercial product, process, or service by trade name, trademark, manufacturer, or otherwise, does not necessarily constitute or imply its endorsement, recommendation, or favoring by the United States Government or any agency thereof or its contractors or subcontractors.

Printed in the United States of America. To obtain copies of this document, contact: Document Control Services, P.O. Box 950, Mailstop H6-08, Richland WA 99352, Phone (509) 372-2420; Fax (509) 376-4989.
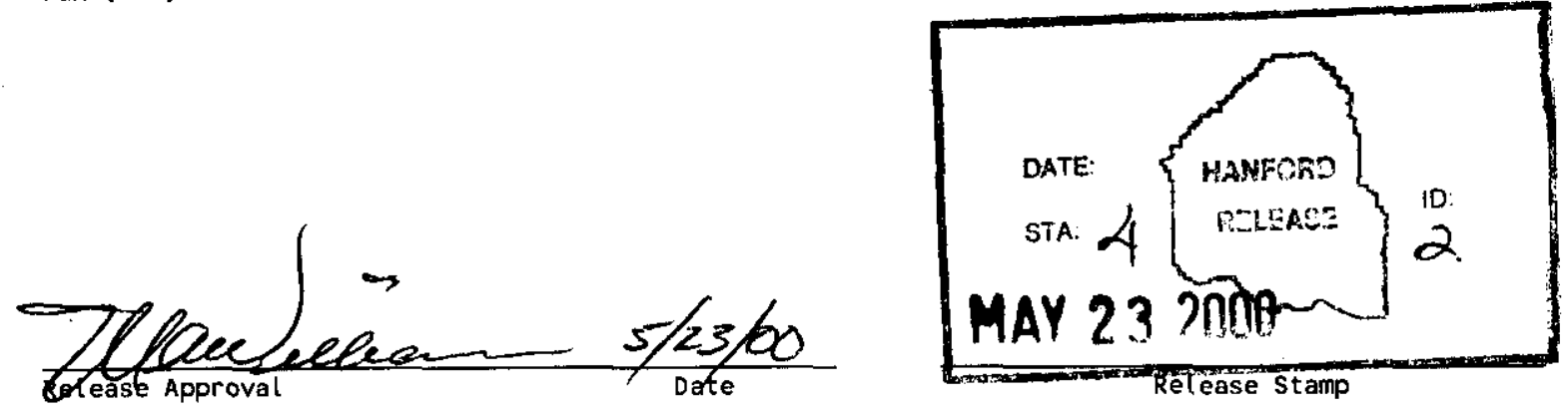

Approved for Public Release 


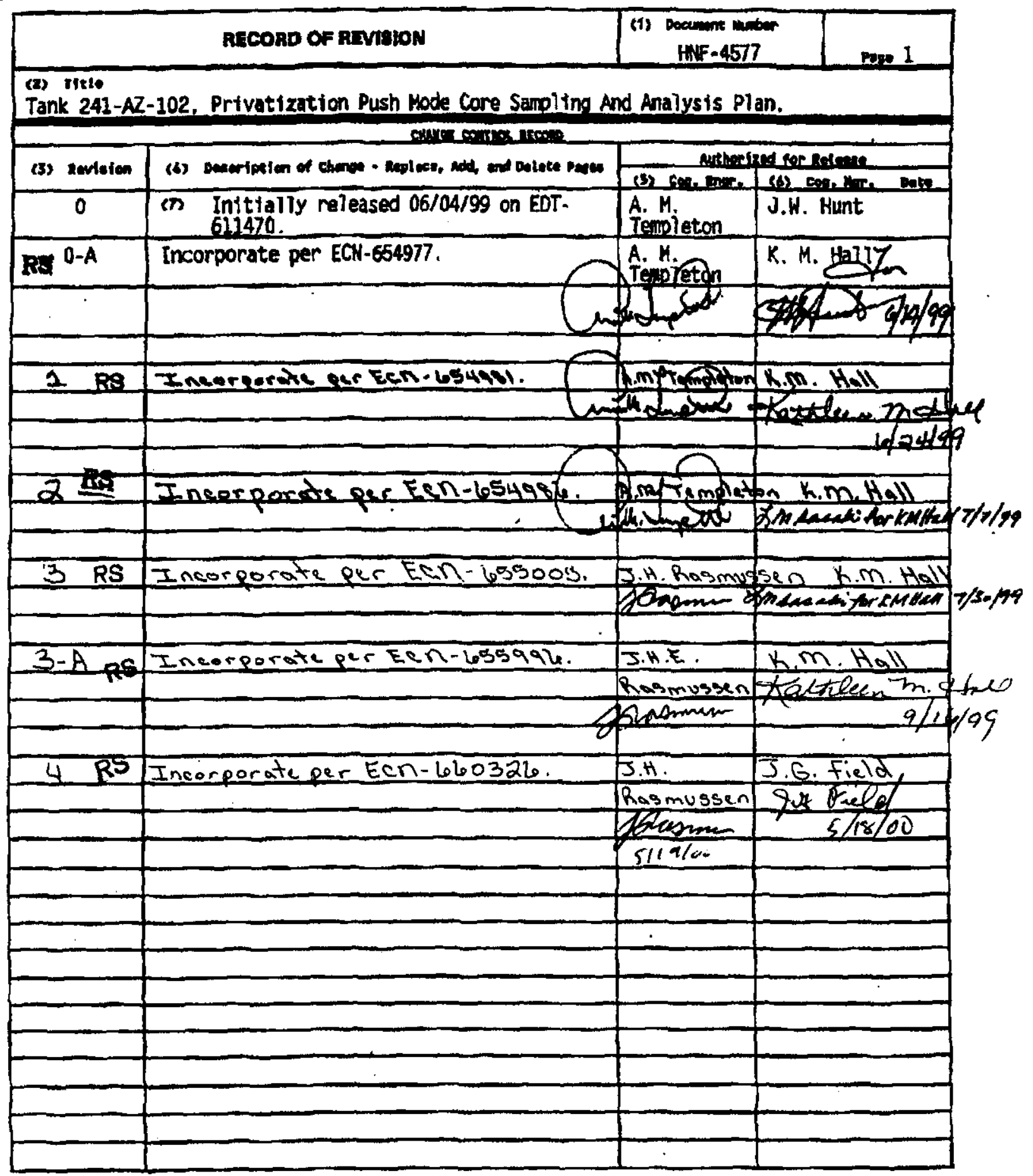

A-75200-005 coessis wertwe 


\section{Tank 241-AZ-102 Privatization Push Mode Core Sampling and Analysis Plan}

J. H. E. Rasmussen

CH2M Hill Hanford Group, Inc.

Prepared for the Office of River Protection 
HNF-4577, Rev. 4

TABLE OF CONTENTS

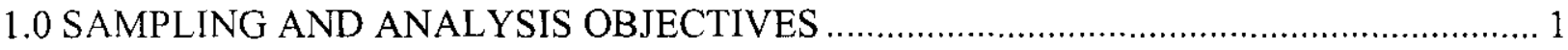

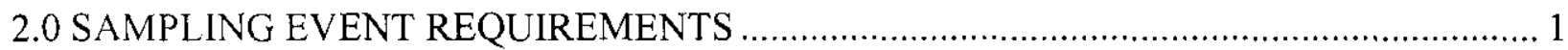

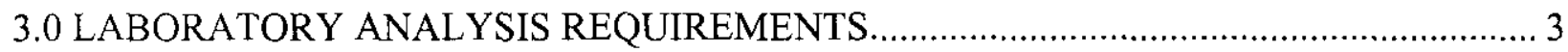

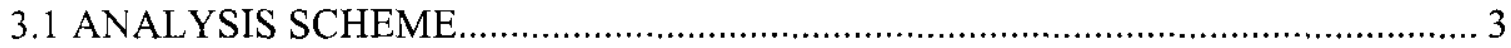

3.2 SPECIFIC METHODS AND ANALYSES...................................................... 4

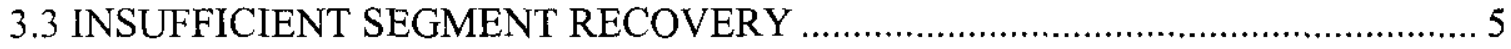

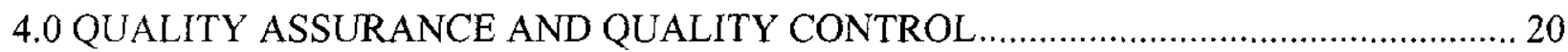

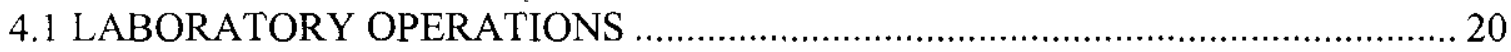

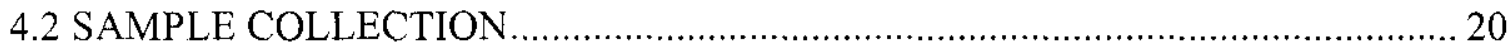

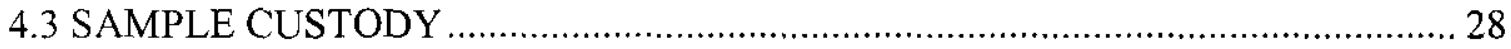

5.0 EXCEPTIONS, CLARIFICATIONS, AND ASSUMPTIONS ……...................................... 28

5.1 EXCEPTIONS TO DATA QUALITY OBJECTIVES REQUIREMENTS ………... 28

5.2 CLARIFICATIONS AND ASSUMPTIONS ……………..................................... 29

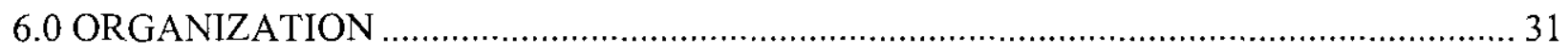

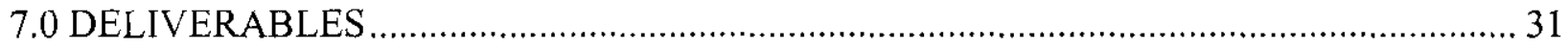

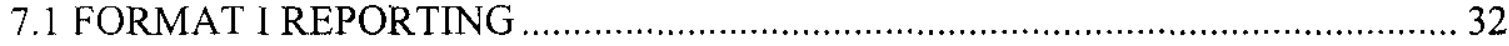

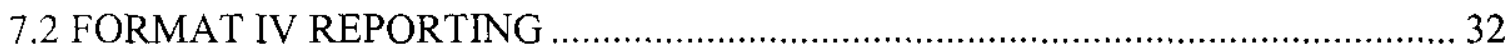

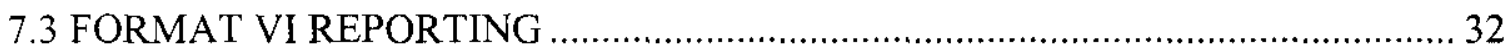

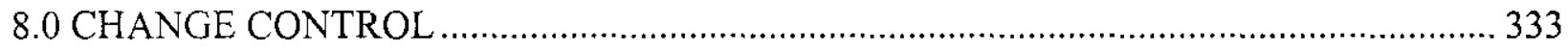

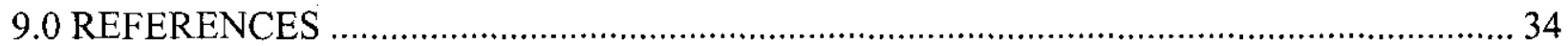

APPENDICES

APPENDIX A: EXPECTED PHYSICAL PROFILE OF TANK 241-AZ-102 CORE

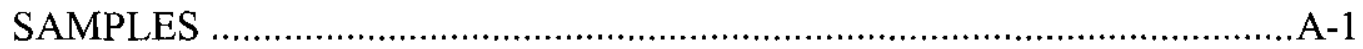

APPENDIX B: 241-AZ-102 WASTE COMPOSITE PREPARATION GUIDELINES ............ B-1 


\section{LIST OF TABLES}

1: Tank 241-AZ-102 Chemical, Radiological, and Physical Analytical Requirements: Liquids...6

2: Tank 241-AZ-102 Chemical, Radiological, and Physical Analytical Requirements: Solids .....9

3: Detection Limits and Minimum Reportable Quantities for LAW Liquids............................ 12

4: Detection Limits and Minimum Reportable Quantities for HLW Solids .............................. 15

5: Quality Control Parameters for Liquid Analyses ....................................................... 21

6: Quality Control Parameters For Solids Analyses ......................................................2 24

7: Tank 241-AZ-102 Project Key Personnel List .........................................................29

A-1: Tank 241-AZ-102 Physical Profile Estimate Risers 15C, 15H, and 24A ......................

\section{LIST OF FIGURES}

A-1: Tank 241-AZ-102 Physical Profile Estimate (Risers 15C, 15H and 24A)...................A-3 


\section{LIST OF ABBREVIATIONS}

\begin{tabular}{|c|c|}
\hline $\mathrm{AEA}$ & alpha energy analysis \\
\hline ALC & air lift circulator \\
\hline AMU & Atomic Mass Unit \\
\hline $\mathrm{Ci}$ & curie \\
\hline $\mathrm{CPO}$ & Characterization Project Operations \\
\hline CVAA & cold vapor atomic absorption \\
\hline DQO & data quality objective \\
\hline DSC & differential scanning calorimetry \\
\hline g & gram \\
\hline $\mathrm{g} / \mathrm{L}$ & gram per liter \\
\hline GEA & gamma energy analysis \\
\hline GPC & gas flow proportional counter \\
\hline HLW & high level waste \\
\hline IC & ion chromatography \\
\hline $\mathrm{ICP} / \mathrm{AES}$ & inductively coupled plasma - atomic emission spectroscopy \\
\hline $\mathrm{ICP} / \mathrm{MS}$ & inductively coupled plasma mass spectroscopy \\
\hline ISE & ion-specific electrode \\
\hline kgal & kilogallon \\
\hline $\mathrm{kL}$ & kiloliter \\
\hline $\mathrm{L}$ & liter \\
\hline LAW & low activity waste \\
\hline LCS & laboratory control standard \\
\hline LFL & lower flammability limit \\
\hline $\mathrm{LiBr}$ & lithium bromide \\
\hline LMHC & Lockheed Martin Hanford Corporation \\
\hline LSC & liquid scintillation \\
\hline MDA & minimum detectable activity \\
\hline $\mathrm{mL}$ & milliliter \\
\hline MRQ & minimum reportable quantity \\
\hline MSL & mean sea level \\
\hline N/A & not applicable \\
\hline NA & not available \\
\hline NCAW & neutralized current acid waste \\
\hline $\mathrm{NHC}$ & Numatec Hanford Corporation \\
\hline ORP & Office of River Protection \\
\hline PIC & person in charge \\
\hline PNNL & Pacific Northwest National Laboratory \\
\hline PRSST & Propagating Reactive System Screening Tool \\
\hline QA & quality assurance \\
\hline QC & quality control \\
\hline RPP & River Protection Project \\
\hline RSD & relative standard deviation \\
\hline RSST & Reactive System Screening Tool \\
\hline
\end{tabular}




\section{HNF-4577, Rev. 4}

SAP

Sep

TBD

TGA

TIMS

TOC

TRU

TWRS

WAPS

WMH

wt $\%$

$\mu \mathrm{Ci}$

$\mu \mathrm{Ci} / \mathrm{g}$

$\mu \mathrm{Ci} / \mathrm{mL}$

$\mu \mathrm{g} / \mathrm{mL}$ sampling and analysis plan

separation required

to be determined

thermogravimetric analysis

thermal ionization mass spectroscopy

total organic carbon

transuranic

Tank Waste Remediation System

Waste Acceptance Product Specifications

Waste Management Hanford

weight percent

microcurie

microcurie per gram

microcuries per milliliter

micrograms per milliliter 


\subsection{SAMPLING AND ANAL YSIS OBJECTIVES}

This sampling and analysis plan (SAP) identifies characterization objectives pertaining to sample collection, laboratory analytical evaluation, and reporting requirements for samples obtained from tank 241-AZ-102. The purpose of this sampling event is to obtain information about the characteristics of the contents of 241-AZ-102 required to satisfy the Data Quality Objectives For TWRS Privatization Phase 1: Confirm Tank T Is An Appropriate Feed Source For High-Level Waste Feed Batch X (HLW DQO) (Nguyen 1999a), Data Quality Objectives For TWRS Privatization Phase 1: Confirm Tank T Is An Appropriate Feed Source For Low-Activity Waste Feed Batch X (LAW DQO) (Nguyen 1999b), Low Activity Waste and High Level Waste Feed Data Quality Objectives (L\&H DQO) (Patello et al. 1999) and Characterization Data Needs for Development, Design, and Operation of Retrieval Equipment Developed through the Data Quality Objective Process (Equipment DQO) (Bloom 1996). The Tank Characterization Technical Sampling Basis document (Brown et al. 1998) indicates that these issues, except the Equipment DQO apply to tank 241-AZ-102 for this sampling event. The Equipment DQO is applied for shear strength measurements of the solids segments only. Poppiti (1999a) requires additional americium-241 analyses of the sludge segments. Poppiti (1999b) directs the shipment of wet Tank 241-AZ-102 sludge samples to the Privatization Contractor. Brown et al. (1998) also identify safety screening and regulatory issues as applicable issues for this tank. However, these issues will not be addressed via this sampling event. Reynolds et al. (1999) concluded that information from previous sampling events was sufficient to satisfy the safety screening requirements for tank 241-AZ-102. Additional analyses of the lowest layer of sludge were requested by Letter of Instruction for 241-AZ-102 Process Test (Jewett 1999) to support retrieval planning. Additional analyses of the lowest layer of sludge were requested by Poppiti $1999 \mathrm{c}$ to identify the contribution of this material to the composition of the tank as a whole.

Push mode core samples will be obtained from risers $15 \mathrm{C}, 15 \mathrm{H}$, and $24 \mathrm{~A}$ to provide sufficient material for the chemical analyses and tests required to satisfy these data quality objectives, and for provision of samples to the Privatization Contractor. The 222-S Laboratory will extrude core samples, composite the liquids and solids, perform chemical analyses, provide subsamples to the Process Chemistry Laboratory, and ship samples to the Privatization Contractor. The Process Chemistry Laboratory will prepare test plans and perform process tests to evaluate the behavior of the 241-AZ-102 waste undergoing the retrieval and treatment scenarios defined in the applicable DQOs. Requirements for analyses of samples originating in the process tests will be documented in the corresponding test plan.

\subsection{SAMPLING EVENT RE QUIREMENTS}

As of June 21, 1999, surveillance readings indicated that tank 241-AZ-102 contained a total waste volume of approximately $3,487 \mathrm{~kL}$ (921 kgal), consisting of 3,091 kL (817 kgal) of supernate and $396 \mathrm{~kL}$ (104 kgal) of sludge (sludge volume per Hanlon 1999). This waste volume is equivalent to 8.51 meters ( 335 inches) of waste as measured from the inside bottom of the tank. A physical profile prediction based on waste fill history and previous sampling information is provided in Appendix A. Previous characterization data indicate that the waste consists primarily of aging waste (Schreiber 1995). Air Lift Circulator (ALC) operation prior to this sampling event may result in suspension of solids in the liquid layer. Because the settling 


\section{HNF-4577, Rev. 4}

behavior of these solids is not well known, the entire liquid layer must be represented by the full core samples.

Prior to core sampling, the dome space (below the riser) shall be measured for the presence of flammable gases. The measurement shall be taken from within the dome space and the data reported as a percentage of the lower flammability limit (LFL). The results shall be transmitted to the tank coordinator within ten working days of the sampling event (Schreiber 1998). If the dome space results are above 10 percent of the LFL when analyzing with a combustible gas meter or above 25 percent of the LFL when analyzing by gas chromatography/mass spectrometry or gas-specific monitoring, the necessity for recurring sampling for the flammable gas concentrations and the frequency of such sampling will be determined by the Flammable Gas Safety Program.

During core sampling, the drill string will be monitored for flammable gas. If the monitoring instrument in the drill string indicates a level greater than $10 \%$ of the LFL during intrusive activities, then a vapor grab sample shall be taken and sent to Pacific Northwest National Laboratory (PNNL) for analysis in accordance with the Flammable Gas DQO. Any additional vapor sampling is not within the scope of this SAP.

Tank 241-AZ-102 will be push mode core sampled using a push or rotary mode core sampling system. Two core samples, consisting of 18 segments each, are expected to be taken from two 6inch diameter risers $15 \mathrm{C}$, and $24 \mathrm{~A}$. The sampling objective is to obtain two full vertical profiles of the waste; therefore, additional segments may need to be taken depending on the accuracy of the current waste volume records in comparison to pre-sampling zip cord readings.

During the sampling event, sludge from riser $15 \mathrm{C}$ has been provided to the Privatization Contractor in order to meet the tight sample shipment schedule required per Poppiti (1999b). Visual observations of segments $1-16$ from risers $15 \mathrm{C}$ and $24 \mathrm{~A}$ indicate that no sludge is suspended in the supernate. Therefore, a third core sample consisting of only segments 16,17 , and 18 will be obtained from riser $15 \mathrm{H}$, and paired with supernate from riser $15 \mathrm{C}$ to constitute the second full core of Tank 241-AZ-102.

Universal zero-flow samplers will be used for these samples. The lower 2 segments of each core shall be $x$-rayed to ensure adequate solids recovery. If quality-affecting changes to the sampling requirements must be made (including the risers, sampling truck, or segments to be sampled), the change must be recorded and approved by the cognizant engineer and tank coordinator before sampling. This information may be recorded on a permanent data sheet or recorded directly in sampling work packages ES-99-00070 for riser 15C, ES-99-00281 for riser 15H, and ES-9900071 for riser $24 \mathrm{~A}$. These work packages contain the operating procedures and the chain-ofcustody records for this sampling event.

One field blank for tank 241-AZ-102 shall be obtained in accordance with procedure TO-060003. The Characterization Project Operations (CPO) person in charge (PIC) or the PIC designate will verify that the field blank is properly created and shipped. For sampling events having multiple PICs, CPO shall determine which PIC will be responsible for the field blank. This field blank is to accompany the samples to the laboratory. All collected samples shall be shipped to 


\section{HNF-4577, Rev. 4}

the laboratory following the Load/Transport Sample Cask(s) procedure (TO-080-090). Core samples should be transported to the laboratory within three calendar days from the time each segment is removed from the tank.

If lithium bromide ( $\mathrm{LiBr}$ ) solution is used in the collection of the core samples, it should be a $0.3 \pm 0.01$ molar solution with a $\mathrm{pH}$ greater than 8 . Characterization Project Operations must state the batch number and amount of fluid added at each segment. This information should be indicated on the chain-of-custody form that accompanies the sample to the laboratory. A sample of the LiBr solution must be provided to the laboratory. This sample shall consist of a container filled with $\mathrm{LiBr}$ solution from the same batch of $\mathrm{LiBr}$ solution used during the sampling. This solution shall be analyzed for lithium and bromide in order to determine the concentration of the tracer at the time the sample was taken. If analysis of the waste samples indicates contamination by the $\mathrm{LiBr}$ solution, these data will be used to determine the amount of contamination. If more than one batch of $\mathrm{LiBr}$ solution is used during sampling event, one solution sample must be provided for each batch in addition to the field blank.

\subsection{LABORATORY ANAL YSIS REQUIREMENTS}

The extrusion, subsampling, compositing, and analysis requirements are described below. For core samples from tank 241-AZ-102, the shipping container must be vented every 27 days to release any accumulated gas.

\subsection{ANALYSIS SCHEME}

Data quality objective requirements drive the analysis of the samples. In order to comply with the LAW, HLW, L\&H, and Equipment DQO, the following steps shall be performed on each sample:

(1) (1) Extrude segments, videotaping the extrusion and photographing the extruded segments. The extrusion procedure is LO-160-103 at the 222-S Laboratory. If the segment contains solids, the solids shall be examined to confirm that layering is not present. If observations indicate that layers are present the cognizant scientist directs subsampling; the segments shall be divided into subsegments to reflect the observed layering.

(2) (2)Analyze each solids segment and subsegment for shear strength, and subsample for particle size analysis before waste is handled further. If observations indicate that layers are present, the cognizant scientist directs subsampling for particle size and shear strength.

(3) (3) Homogenize solids segments and subsegments. Take three subsamples of each solids segment and subsegment and analyze for ${ }^{241}$ Am per Poppiti (1999a). Analyze for total Alpha, solids density and \% wt solids by gravimetry, weight fraction of centrifuged solids and centrifuged solids density.

(4) Allow drainable liquid samples to settle for at least 16 hours. Record volume percent solids, and note any evidence of gas releases or separated liquid phases. 


\section{HNF-4577, Rev. 4}

(5) Subsample individual segments for analysis per Tables 1 and 2.

(6) Prepare separate solids composites for each riser as directed by tank coordinator per the guidelines in Appendix B.

(7) Provide a portion of each riser solids composite and supernate to Process Chemistry for solubility screening testing per Person 1999 . For the riser $15 \mathrm{H}$ composite, supernate from riser $15 \mathrm{C}$ will be paired with the sludge from riser $15 \mathrm{H}$.

(8) Provide a portion of each riser solids composite and supernate to Process Chemistry for Retrieval testing per Herting 1999. For the riser $15 \mathrm{H}$ composite, supernate from riser $15 \mathrm{C}$ will be paired with the sludge from riser $15 \mathrm{H}$.

(9) Combine a subsample of each riser composite with the representative amount of supernate as determined by tank coordinator. For the riser $15 \mathrm{H}$ composite, supernate from riser $15 \mathrm{C}$ will be paired with the sludge from riser $15 \mathrm{H}$.

(10) Mix solids and liquids and allow to settle for at least 16 hours. Record weight, volume, and volume \% settled solids for each composite, and note any evidence of floating layers (organics or solids) or gas generation.

(11) Separate each riser composite by centrifugation. The responsible chemist shall ensure that the centrifuged solids contain no separate liquid phase.

(12) Analyze 3 subsamples of solid fraction of each riser composite separated in step 11 per Table 2.

(13) Analyze 3 subsamples of liquid fraction of each riser composite separated in step 11 per Table 1.

(14) Archive remaining sample material from individual segments and composites.

If liner liquid is observed during extrusion and the liquid is in sufficient quantity to collect, the liner liquid may be retained and analyzed at the discretion of the tank coordinator. In this event, this addition of analyses may not require a revision to this SAP.

Opportunistic analyses as defined in Kristofzski (1996) are to be included when the laboratory is not operating at maximum capacity. Any decisions, observations, or deviations made to this work plan, or during the sample breakdown and analyses shall be documented in writing with justification. These decisions and observations shall be reported in the data report. The reporting formats for analyses are contained in Tables 1 and 2 and are described in Section 7.0.

\subsection{SPECIFIC METHODS AND ANALYSES}

The analyses in Tables 1 and 2 to be performed on the tank 241-AZ-102 core samples are based on the HLW, LAW, L\&H, and Equipment DQOs. The laboratory procedure numbers which shall be used for the analyses are included in the tables. Sample preparation procedures that may be used at the 222-S Laboratory are LA-549-141 for fusion digestion, LA-505-159 or LA-505-163 for acid digestion of samples, and LA-504-101 for water leach of solids. The laboratory is to notify the tank coordinator once analyses are complete.

Duplication of effort shall be avoided where practical. For example, many of the analyses required for the L\&H DQO are also required by the HLW and LAW DQOs. If process testing per the HLW and LAW DQOs determines that dilution is not required for waste retrieval, analyses per the L\&H DQO can meet the corresponding data needs of the HLW and LAW 


\section{HNF-4577, Rev. 4}

DQOs. However, if process testing determines that dilution of the waste is required, then separate analyses of the diluted samples will be as specified in the test plan.

The HLW, LAW, and L\&H DQOs specify Minimum Reportable Quantities (MRQs) of those analytes listed in Tables 1 and 2 . The laboratory is to notify the tank coordinator of any circumstances which prevent achieving detection limits at or below the MRQ for any analyte, and recommend a corrective course of action.

The L\&H DQO requires that a material balance be performed for all material sampled and composited as part of this sampling event. Weights of all liquids and solids subsampled shall be reported in the Format IV data package.

\subsection{INSUFFICIENT SEGM ENT RECOVERY}

If the amount of material recovered from samples taken from 241-AZ-102 is insufficient to form the composites and perform the analyses requested in the SAP and permit a minimum $100 \mathrm{~mL}$ archive per sample, the laboratory shall notify the tank coordinator within one working day. At that time, a prioritization of the analyses and/or compositing scheme may be provided to the laboratory. Any analyses prescribed by the SAP, but not performed, shall be identified in the appropriate data report with justification provided for nonperformance. 


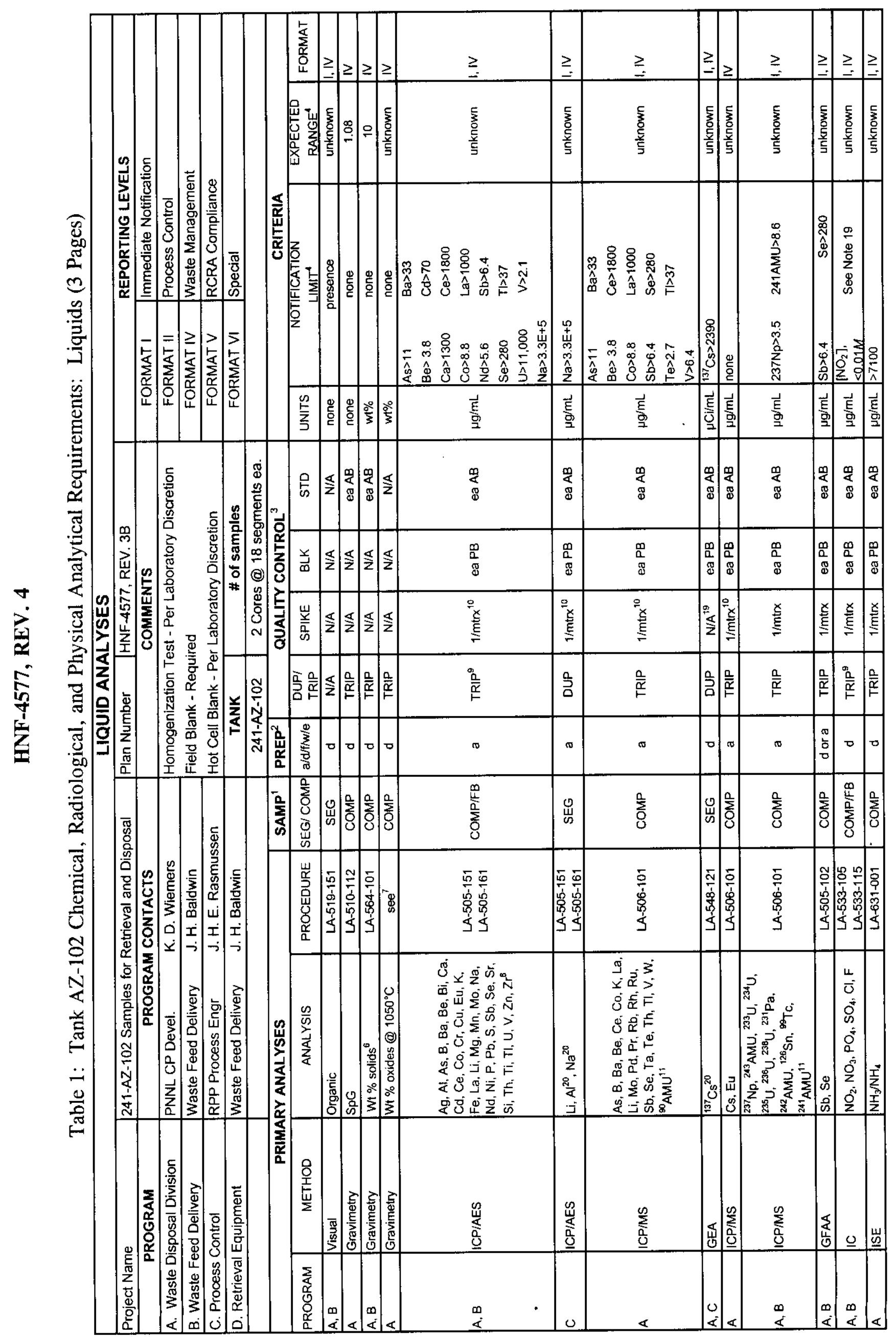




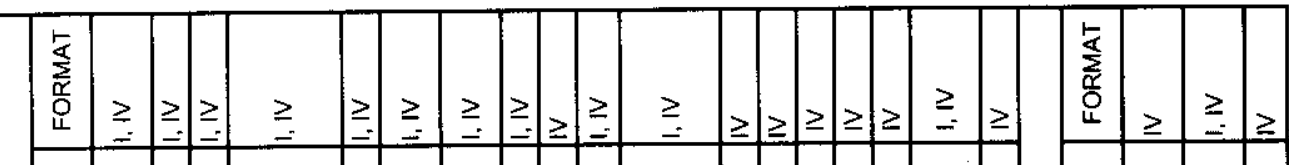

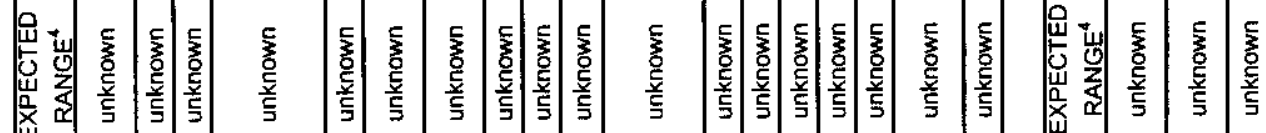

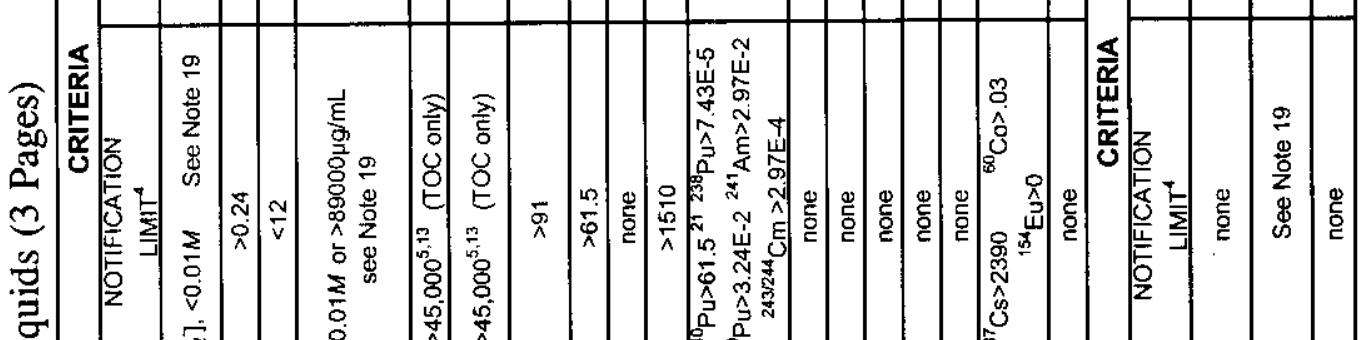

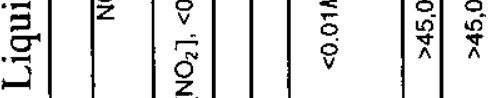

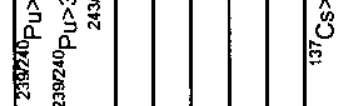

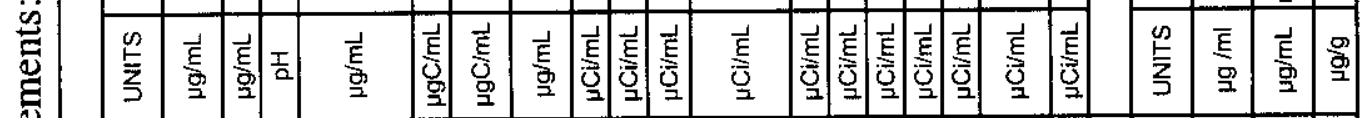

.

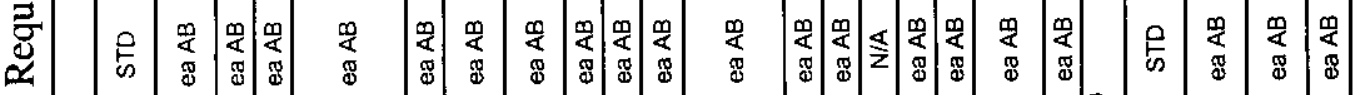

胥

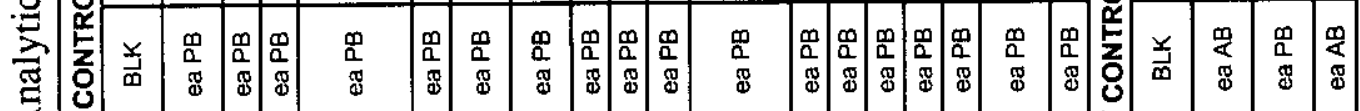

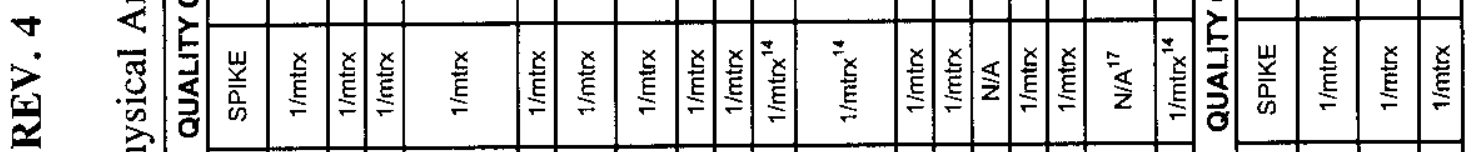

in

을

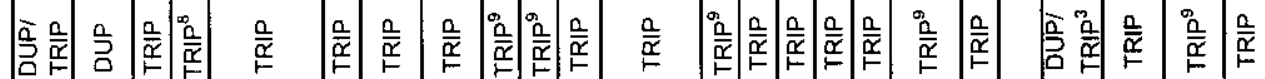

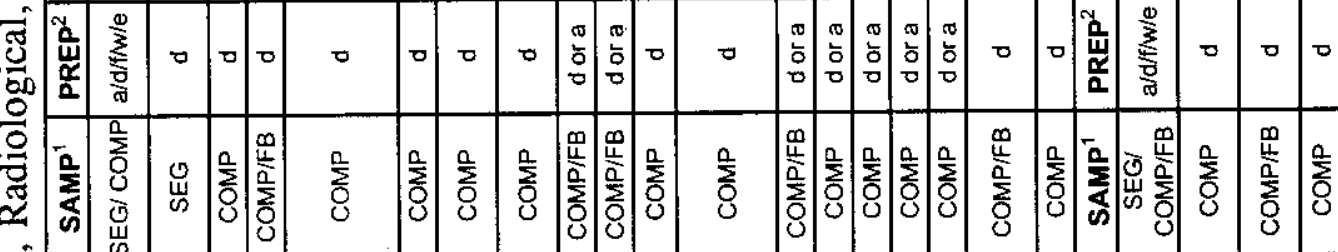

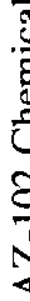

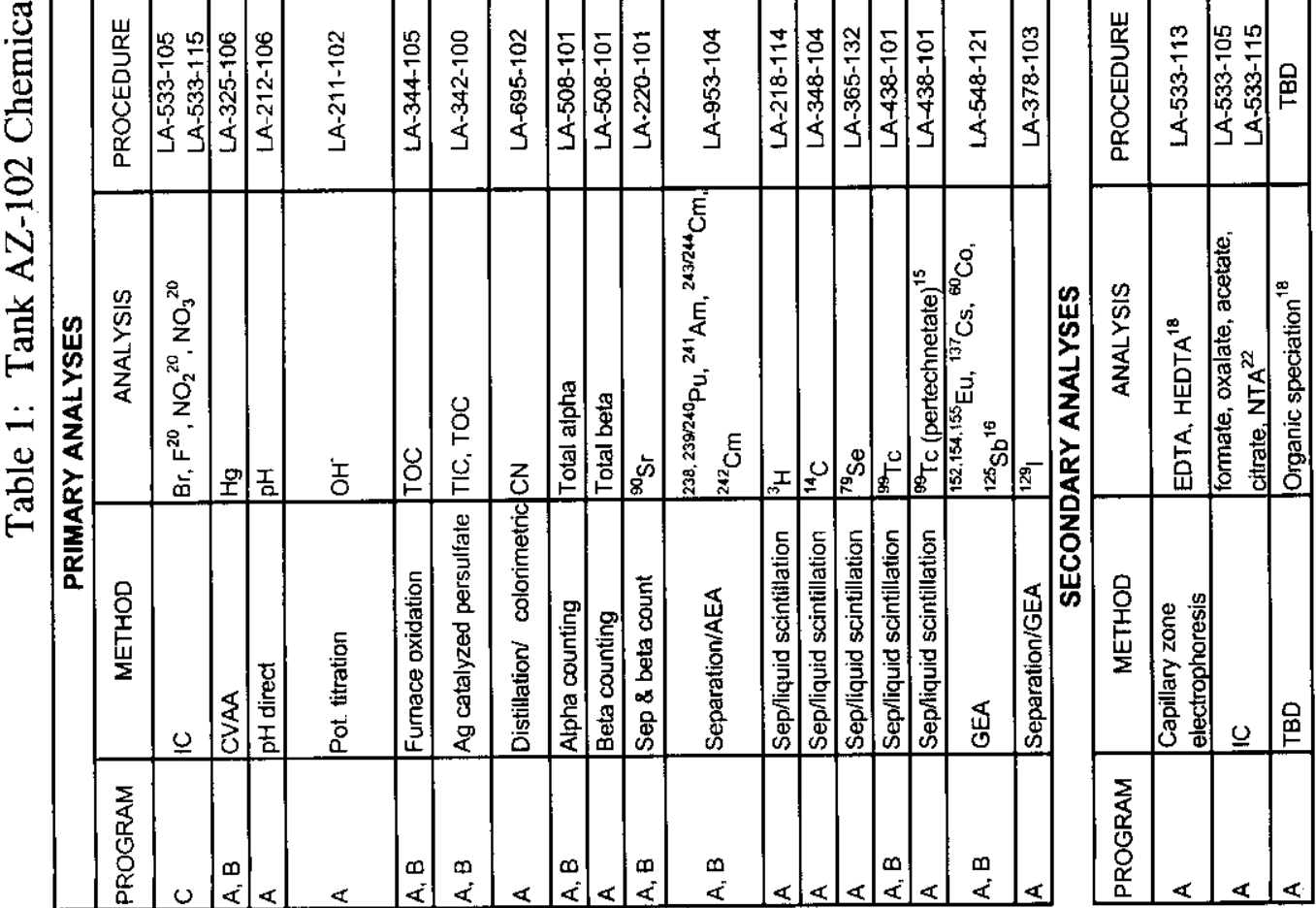

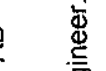

产

兽

复 $\frac{\text { a }}{\overline{0}}$

:

$\stackrel{9}{\frac{9}{2}}$

i

(1)

용

동 


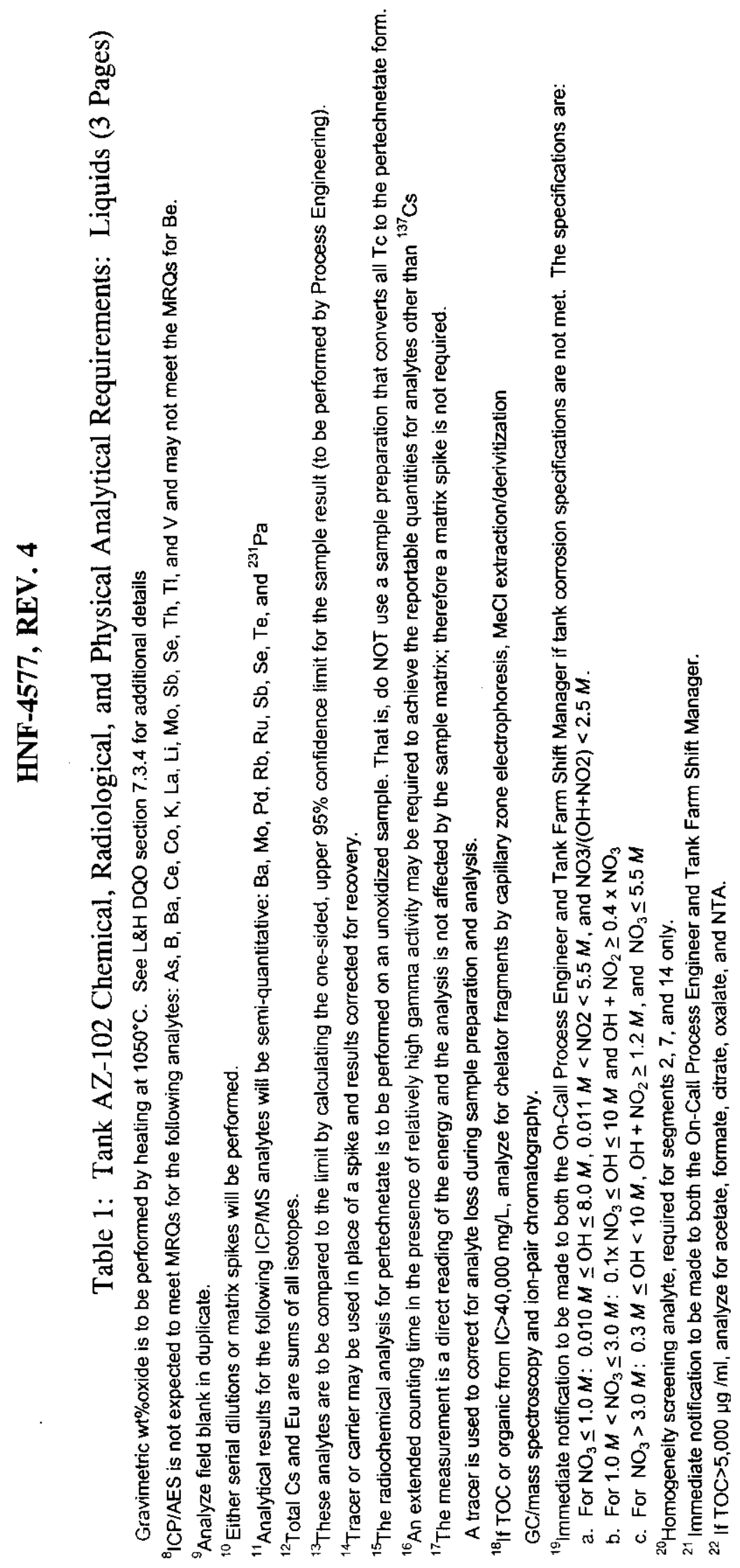




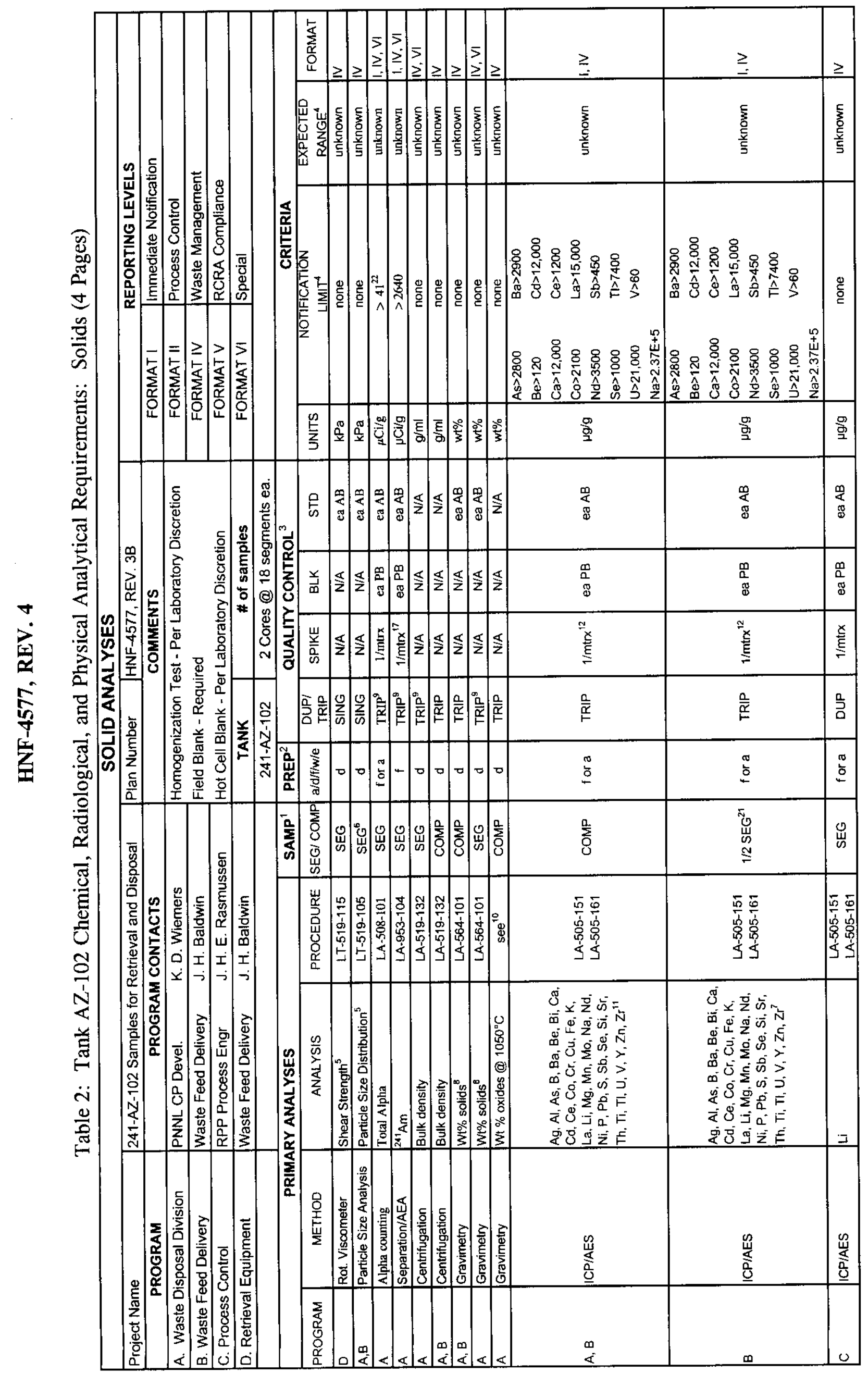




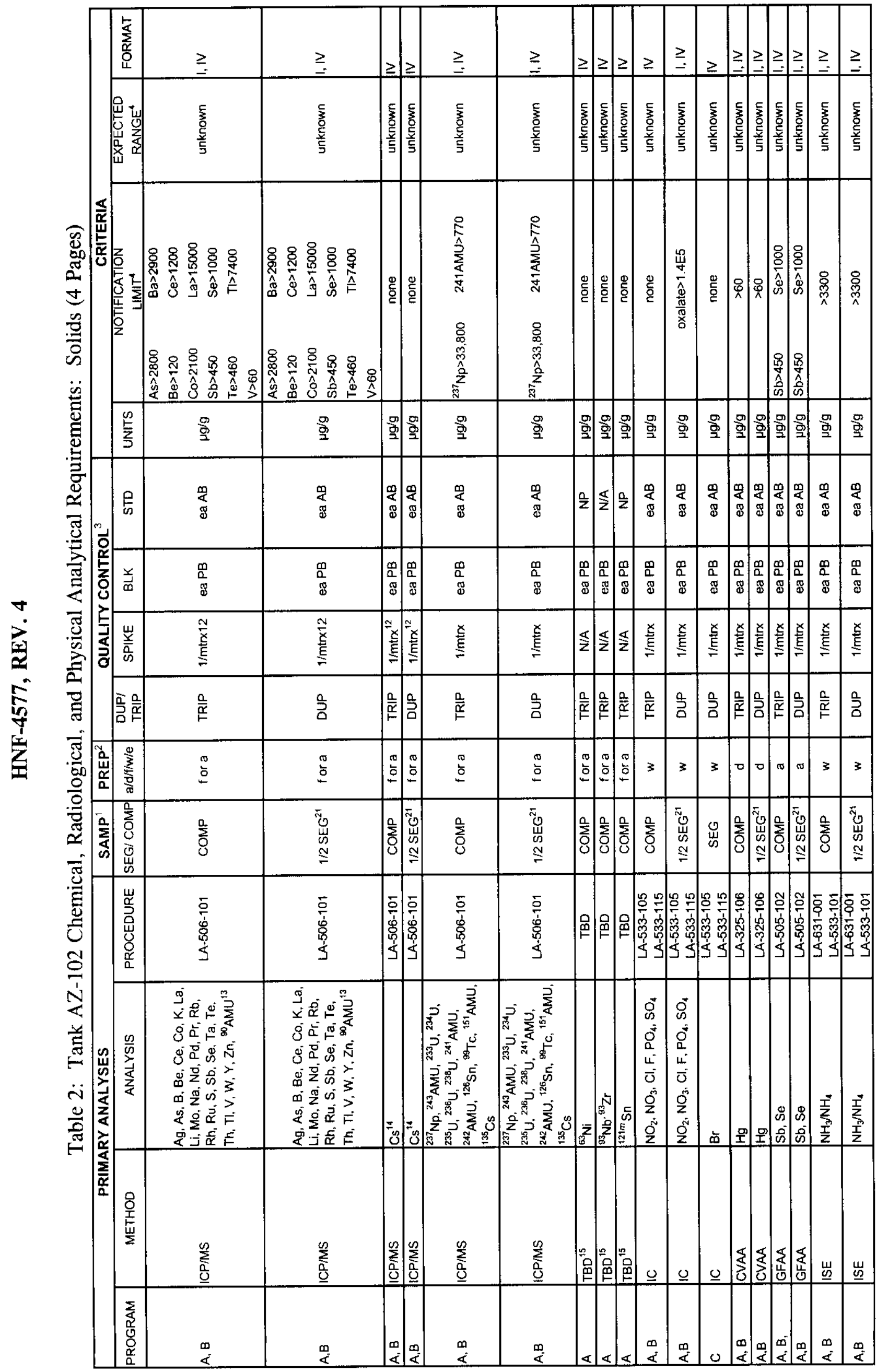




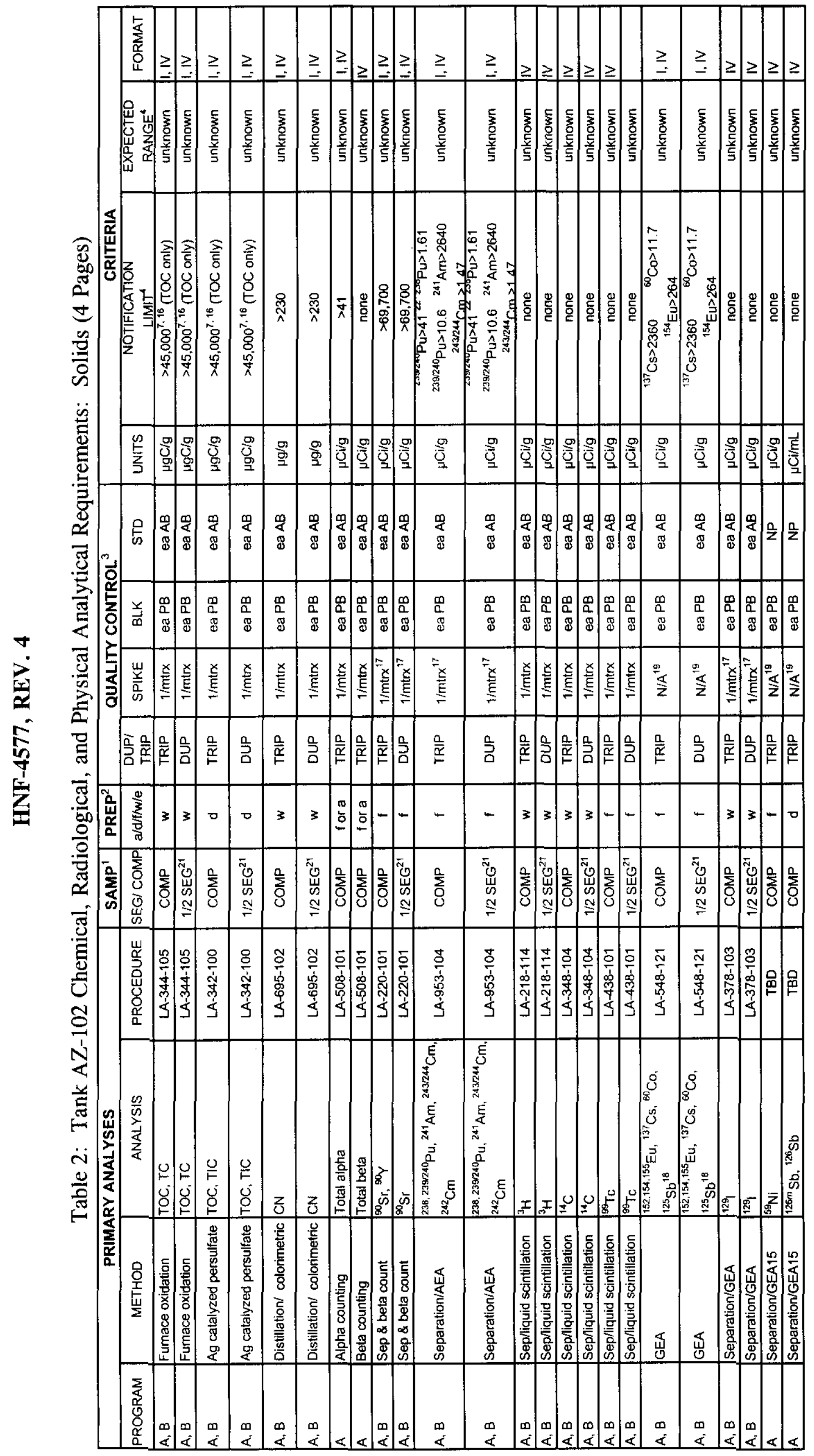




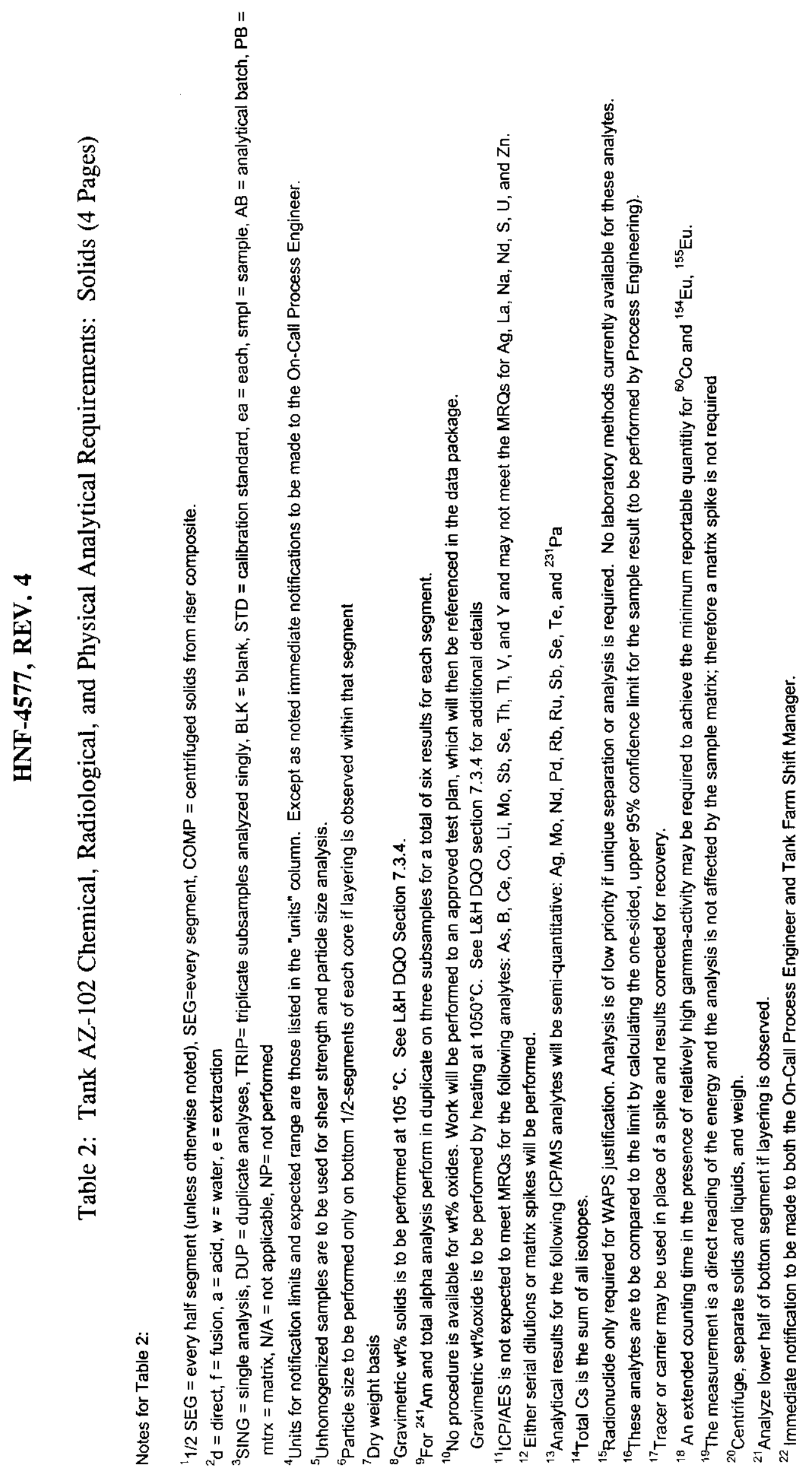


Table 3. Detection Limits and Minimum Reportable Quantities for LAW Liquids (3 Sheets)

\begin{tabular}{|c|c|c|c|c|}
\hline Analyte & Method & $\begin{array}{c}\text { Estimated Quantitation } \\
\text { Limit/Minimum Detectable } \\
\text { Activity }^{(1)}\end{array}$ & $\begin{array}{c}\text { Minimum Reportable Quantity } \\
\text { (MRQ) }^{(1)}\end{array}$ & Units \\
\hline $\mathrm{Ag}$ & ICP/AES & $5.5 \mathrm{E}+00$ & $1.7 \mathrm{E}+01$ & $\mu \mathrm{g} / \mathrm{mL}$ \\
\hline $\mathrm{Al}$ & ICP/AES & $2.5 \mathrm{E}+01$ & $7.5 \mathrm{E}+01$ & $\mu \mathrm{g} / \mathrm{mL}$ \\
\hline As & $\mathrm{ICP} / \mathrm{MS}$ & $7.5 \mathrm{E}-01(1.0 \mathrm{E}+00)$ & $2.3 \mathrm{E}+00(3.0 \mathrm{E}+00)$ & $\mu \mathrm{g} / \mathrm{mL}$ \\
\hline B & ICP/MS & $7.5 \mathrm{E}-01$ & $2.3 \mathrm{E}+00$ & $\mu \mathrm{g} / \mathrm{mL}$ \\
\hline $\mathrm{Ba}$ & $\mathrm{ICP} / \mathrm{MS}$ & $7.5 \mathrm{E}-01(2.6 \mathrm{E}+01)$ & $2.3 \mathrm{E}+00(7.8 \mathrm{E}+01)$ & $\mu \mathrm{g} / \mathrm{mL}$ \\
\hline $\mathrm{Be}$ & ICP/MS & $7.5 \mathrm{E}-01(3.0 \mathrm{E}+00)$ & $2.3 \mathrm{E}+00(9.9 \mathrm{E}+00)$ & $\mu \mathrm{g} / \mathrm{mL}$ \\
\hline $\mathrm{Bi}$ & ICP/AES & $5.5 \mathrm{E}+01$ & $1.7 \mathrm{E}+02$ & $\mu \mathrm{g} / \mathrm{mL}$ \\
\hline $\mathrm{Ca}$ & $\mathrm{ICP} / \mathrm{AES}$ & $5.0 \mathrm{E}+01$ & $1.5 \mathrm{E}+02$ & $\mu \mathrm{g} / \mathrm{mL}$ \\
\hline $\mathrm{Cd}$ & ICP/AES & $2.5 \mathrm{E}+00$ & $7.5 \mathrm{E}+00$ & $\mu \mathrm{g} / \mathrm{mL}$ \\
\hline $\mathrm{Ce}$ & ICP/MS & $7.5 \mathrm{E}-01$ & $2.3 \mathrm{E}+00$ & $\mu \mathrm{g} / \mathrm{mL}$ \\
\hline $\mathrm{Cr}$ & ICP/AES & $5.0 \mathrm{E}+00$ & $1.5 \mathrm{E}+01$ & $\mu \mathrm{g} / \mathrm{mL}$ \\
\hline $\mathrm{Cu}$ & ICP/AES & $5.5 \mathrm{E}+00$ & $1.7 \mathrm{E}+01$ & $\mu \mathrm{g} / \mathrm{mL}$ \\
\hline Cs, total & ICP/MS & $5.0 \mathrm{E}-01$ & $1.5 \mathrm{E} 00$ & $\mu \mathrm{g} / \mathrm{mL}$ \\
\hline Eu, total & $\mathrm{ICP} / \mathrm{MS}$ & $2.0 \mathrm{E}+01$ & $6.0 \mathrm{E}+01$ & $\mu \mathrm{g} / \mathrm{mL}$ \\
\hline $\mathrm{Fe}$ & ICP/AES & $2.5 \mathrm{E}+01(3.0 \mathrm{E}+01)$ & $7.5 \mathrm{E}+01(9.9 \mathrm{E}+01)$ & $\mu \mathrm{g} / \mathrm{mL}$ \\
\hline $\mathrm{Hg}$ & CVAA & $5.0 \mathrm{E}-01$ & $1.5 \mathrm{E}+00(2.0 \mathrm{E}+00)$ & $\mu \mathrm{g} / \mathrm{mL}$ \\
\hline $\mathrm{K}$ & ICP/AES & $2.5 \mathrm{E}+02$ & $7.5 \mathrm{E}+02(7.5 \mathrm{E}+01)$ & $\mu \mathrm{g} / \mathrm{mL}$ \\
\hline La. & ICP/MS & $7.5 \mathrm{E}-01(2.5 \mathrm{E}+01)$ & $2.3 \mathrm{E}+00(3.5 \mathrm{E}+01)$ & $\mu \mathrm{g} / \mathrm{mL}$ \\
\hline $\mathrm{Li}$ & ICP/MS & $7.5 \mathrm{E}-01$ & $2.3 \mathrm{E}+00$ & $\mu \mathrm{g} / \mathrm{mL}$ \\
\hline $\mathrm{Mg}$ & ICP/AES & $5.5 \mathrm{E}+01 \quad(5.0 \mathrm{E}+01)$ & $1.7 \mathrm{E}+02(1.5 \mathrm{E}+02)$ & $\mu \mathrm{g} / \mathrm{mL}$ \\
\hline $\mathrm{Mn}$ & ICP/AES & $5.5 \mathrm{E}+00$ & $1.7 \mathrm{E}+01$ & $\mu \mathrm{g} / \mathrm{mL}$ \\
\hline Mo & ICP/MS & $7.5 \mathrm{E}-01(3.0 \mathrm{E}+01)$ & $2.3 \mathrm{E}+00(9.0 \mathrm{E}+01)$ & $\mu \mathrm{g} / \mathrm{mL}$ \\
\hline $\mathrm{Na}$ & ICP/AES & $5.5 \mathrm{E}+01$ & $1.7 \mathrm{E}+02$ & $\mu \mathrm{g} / \mathrm{mL}$ \\
\hline $\mathrm{Nd}$ & ICP/AES & $5.5 \mathrm{E}+01$ & $1.7 \mathrm{E}+02$ & $\mu \mathrm{g} / \mathrm{mL}$ \\
\hline $\mathrm{Ni}$ & ICP/AES & $1.0 \mathrm{E}+01$ & $3.0 \mathrm{E}+01$ & $\mu \mathrm{g} / \mathrm{mL}$ \\
\hline$P$ & ICP/AES & $1.1 \mathrm{E}+02$ & $3.3 \mathrm{E}+02$ & $\mu \mathrm{g} / \mathrm{mL}$ \\
\hline $\mathrm{Pb}$ & ICP/AES & $9.9 \mathrm{E}+01$ & $3.0 \mathrm{E}+02$ & $\mu \mathrm{g} / \mathrm{mL}$ \\
\hline $\mathrm{Pd}$ & ICP/AES & $1.3 \mathrm{E}+02$ & $3.9 \mathrm{E}+02$ & $\mu \mathrm{g} / \mathrm{mL}$ \\
\hline $\mathrm{Pr}$ & ICP/MS & $7.5 \mathrm{E}-01$ & $2.3 \mathrm{E}+00$ & $\mu \mathrm{g} / \mathrm{mL}$ \\
\hline $\mathrm{Pt}$ & ICP/AES & $\mathrm{NS}^{2}(6.0 \mathrm{E}-01)$ & $\mathrm{NS}^{2}(1.8 \mathrm{E}+00)$ & $\mu \mathrm{g} / \mathrm{mL}$ \\
\hline $\mathrm{Rb}$ & ICP/MS & $7.5 \mathrm{E}-01$ & $2.3 \mathrm{E}+00$ & $\mu \mathrm{g} / \mathrm{mL}$ \\
\hline $\mathrm{Rh}$ & ICP/AES & $6.0 \mathrm{E}+00$ & $1.8 \mathrm{E}+01$ & $\mu \mathrm{g} / \mathrm{mL}$ \\
\hline $\mathrm{Ru}$ & ICP/AES & $1.2 \mathrm{E}+01$ & $3.6 \mathrm{E}+01$ & $\mu \mathrm{g} / \mathrm{mL}$ \\
\hline$S$ & ICP/AES & $5.5 \mathrm{E}+01$ & $1.7 \mathrm{E}+02$ & $\mu \mathrm{g} / \mathrm{mL}$ \\
\hline $\mathrm{Sb}$ & ICP/MS & $7.5 \mathrm{E}-01(3.5 \mathrm{E}+01)$ & $2.3 \mathrm{E}+00(1.0 \mathrm{E}+02)$ & $\mu \mathrm{g} / \mathrm{mL}$ \\
\hline $\mathrm{Se}$ & ICP/MS & $7.5 \mathrm{E}-01(5.5 \mathrm{E}+01)$ & $2.3 \mathrm{E}+00(1.7 \mathrm{E}+02)$ & $\mu \mathrm{g} / \mathrm{mL}$ \\
\hline $\mathrm{Si}$ & ICP/AES & $3.0 \mathrm{E}+01$ & $9.0 \mathrm{E}+01$ & $\mu \mathrm{g} / \mathrm{mL}$ \\
\hline Sn & ICP/AES & $\mathrm{NS}^{2}(5.0 \mathrm{E}+02)$ & $\mathrm{NS}^{2}(1.5 \mathrm{E}+03)$ & $\mu \mathrm{g} / \mathrm{mL}$ \\
\hline
\end{tabular}


HNF-4577, Rev. 4

Table 3. Detection Limits and Minimum Reportable Quantities for LAW Liquids (3 Sheets)

\begin{tabular}{|c|c|c|c|c|}
\hline Analyte & Method & $\begin{array}{c}\text { Estimated Quantitation } \\
\text { Limit/Minimum Detectable } \\
\text { Activity }^{(1)}\end{array}$ & $\begin{array}{c}\text { Minimum Reportable Quantity } \\
(\mathrm{MRQ})^{(1)}\end{array}$ & Units \\
\hline $\mathrm{Sr}$ & ICP/AES & $5.5 \mathrm{E}+00$ & $1.7 \mathrm{E}+01$ & $\mu \mathrm{g} / \mathrm{mL}$ \\
\hline $\mathrm{Ta}$ & ICP/MS & $7.5 \mathrm{E}-01$ & $2.3 \mathrm{E}+00$ & $\mu \mathrm{g} / \mathrm{mL}$ \\
\hline $\mathrm{Te}$ & ICP/MS & $7.5 \mathrm{E}-01$ & $2.3 \mathrm{E}+00$ & $\mu \mathrm{g} / \mathrm{mL}$ \\
\hline $\mathrm{Th}$ & ICP/MS & $7.5 \mathrm{E}-01$ & $2.3 \mathrm{E}+00$ & $\mu \mathrm{g} / \mathrm{mL}$ \\
\hline $\mathrm{Ti}$ & ICP/AES & $5.5 \mathrm{E}+00$ & $1.7 \mathrm{E}+01$ & $\mu \mathrm{g} / \mathrm{mL}$ \\
\hline $\mathrm{Tl}$ & ICP/MS & $7.5 \mathrm{E}-01$ & $2.3 \mathrm{E}+00$ & $\mu \mathrm{g} / \mathrm{mL}$ \\
\hline $\mathrm{U}$ & ICP/AES & $2.60 \mathrm{E}+02(2.0 \mathrm{E}-01)$ & $7.8 \mathrm{E}+02(6.0 \mathrm{E}+02)$ & $\mu \mathrm{g} / \mathrm{mL}$ \\
\hline $\mathrm{V}$ & ICP/MS & $7.5 \mathrm{E}-01(3.0 \mathrm{E}+01)$ & $2.3 \mathrm{E}+00(9.0 \mathrm{E}+01)$ & $\mu \mathrm{g} / \mathrm{mL}$ \\
\hline W & $\mathrm{ICP} / \mathrm{MS}$ & $7.5 \mathrm{E}-01$ & $2.3 \mathrm{E}+00$ & $\mu \mathrm{g} / \mathrm{mL}$ \\
\hline $\mathrm{Y}\left({ }^{90} \mathrm{AMU}\right)$ & ICP/MS & $7.5 \mathrm{E}-01(1.0 \mathrm{E}+02)$ & $2.3 \mathrm{E}+00(2.0 \mathrm{E}+02)$ & $\mu \mathrm{g} / \mathrm{mL}$ \\
\hline $\mathrm{Y}$ & ICP/AES & $\mathrm{NS}^{2}(1.0 \mathrm{E}+02)$ & $\mathrm{NS}^{2}(2.0 \mathrm{E}+02)$ & $\mu \mathrm{g} / \mathrm{mL}$ \\
\hline $\mathrm{Zn}$ & ICP/AES & $5.5 \mathrm{E}+00$ & $1.7 \mathrm{E}+01$ & $\mu \mathrm{g} / \mathrm{mL}$ \\
\hline $\mathrm{Zr}$ & ICP/AES & $5.5 \mathrm{E}+00$ & $1.7 \mathrm{E}+01$ & $\mu \mathrm{g} / \mathrm{mL}$ \\
\hline${ }^{3} \mathrm{H}$ & LSC & $7.0 \mathrm{E}-03$ & $2.1 \mathrm{E}-02$ & $\mu \mathrm{Ci} / \mathrm{mL}$ \\
\hline${ }^{14} \mathrm{C}$ & LSC & $2.4 \mathrm{E}-04$ & $7.2 \mathrm{E}-04$ & $\mu \mathrm{Ci} / \mathrm{mL}$ \\
\hline${ }^{60} \mathrm{Co}^{(3)}$ & GEA & $7.0 \mathrm{E}-04$ & $2.1 \mathrm{E}-03$ & $\mu \mathrm{Ci} / \mathrm{mL}$ \\
\hline${ }^{79} \mathrm{Se}$ & LSC & $3.0 \mathrm{E}-05$ & $9.0 \mathrm{E}-05$ & $\mu \mathrm{Ci} / \mathrm{mL}$ \\
\hline${ }^{89} \mathrm{Sr},{ }^{90} \mathrm{Sr}$ & beta count & $1.0 \mathrm{E}-02(5.0 \mathrm{E}-02)$ & $3.0 \mathrm{E}-02(1.5 \mathrm{E}-01)$ & $\mu \mathrm{Ci} / \mathrm{mL}$ \\
\hline${ }^{99} \mathrm{Tc}$ (total) & ICP/MS & $5.0 \mathrm{E}-04$ & $1.5 \mathrm{E}-03$ & $\mu \mathrm{Ci} / \mathrm{mL}$ \\
\hline $\begin{array}{l}{ }^{99} \mathrm{Tc} \text { (pertech- } \\
\text { netate) }\end{array}$ & TBD & TBD & TBD & $\mu \mathrm{Ci} / \mathrm{mL}$ \\
\hline${ }^{125} \mathrm{Sb}$ & GEA & $5.6 \mathrm{E}-01$ & $1.7 \mathrm{E}+00$ & $\mu \mathrm{Ci} / \mathrm{mL}$ \\
\hline${ }^{126} \mathrm{Sn}$ & ICP/MS & $2.0 \mathrm{E}-03$ & $6.0 \mathrm{E}-03$ & $\mu \mathrm{Ci} / \mathrm{mL}$ \\
\hline 129 & GEA & $5.8 \mathrm{E}-06(3.5 \mathrm{E}-04)$ & $1.8 \mathrm{E}-05(1.1 \mathrm{E}-03)$ & $\mu \mathrm{Ci} / \mathrm{mL}$ \\
\hline${ }^{137} \mathrm{Cs}$ & GEA & $1.3 \mathrm{E}-01(3.0 \mathrm{E}+00)$ & $3.9 \mathrm{E}-01(9.0 \mathrm{E}+00)$ & $\mu \mathrm{Ci} / \mathrm{mL}$ \\
\hline${ }^{152} \mathrm{Eu}^{(3)}$ & GEA & TBD & TBD & $\mu \mathrm{Ci} / \mathrm{mL}$ \\
\hline${ }^{154} \mathrm{Eu}^{(3)}$ & GEA & $6.5 \mathrm{E}-03$ & $2.0 \mathrm{E}-02(2.0 \mathrm{E}-03)$ & $\mu \mathrm{Ci} / \mathrm{mL}$ \\
\hline${ }^{155} \mathrm{Eu}^{(3)}$ & GEA & $3.0 \mathrm{E}-02$ & $9.0 \mathrm{E}-02$ & $\mu \mathrm{Ci} / \mathrm{mL}$ \\
\hline${ }^{231} \mathrm{~Pa}$ & ICP/MS & TBD & TBD & $\mu \mathrm{Ci} / \mathrm{mL}$ \\
\hline${ }^{233} \mathrm{U}$ & ICP/MS & $1.4 \mathrm{E}-04(6.0 \mathrm{E}-04)$ & 4.2E-04 (1.8E-03) & $\mu \mathrm{Ci} / \mathrm{mL}$ \\
\hline${ }^{234} \mathrm{U}$ & ICP/MS & 4.4E-05 (4.0E-08) & $1.2 \mathrm{E}-04(1.2 \mathrm{E}-07)$ & $\mu \mathrm{Ci} / \mathrm{mL}$ \\
\hline${ }^{235} \mathrm{U}$ & $\mathrm{ICP} / \mathrm{MS}$ & $1.5 \mathrm{E}-08(1.1 \mathrm{E}-06)$ & $4.5 \mathrm{E}-08(4.5 \mathrm{E}-08)$ & $\mu \mathrm{Ci} / \mathrm{mL}$ \\
\hline${ }^{236} \mathrm{U}$ & $\mathrm{ICP} / \mathrm{MS}$ & $4.5 \mathrm{E}-07$ & $1.4 \mathrm{E}-06$ & $\mu \mathrm{Ci} / \mathrm{mL}$ \\
\hline${ }^{237} \mathrm{~Np}$ & $\mathrm{ICP} / \mathrm{MS}$ & $1.3 \mathrm{E}-05(9.1 \mathrm{E}-03)$ & $3.9 \mathrm{E}-05(2.7 \mathrm{E}-02)$ & $\mu \mathrm{Ci} / \mathrm{mL}$ \\
\hline${ }^{238} \mathrm{Pu}$ & AEA & 3.4E-03 (3.2E-03) & $1.0 \mathrm{E}-02(9.6 \mathrm{E}-03)$ & $\mu \mathrm{Ci} / \mathrm{mL}$ \\
\hline${ }^{238} \mathrm{U}$ & ICP/MS & $2.4 \mathrm{E}-09(1.7 \mathrm{E}-07)$ & 7.2E-09 (5.0E-07) & $\mu \mathrm{Ci} / \mathrm{mL}$ \\
\hline${ }^{239} \mathrm{Pu}$ & $\mathrm{AEA}$ & $3.4 \mathrm{E}-03(3.20 \mathrm{E}-03)$ & $1.0 \mathrm{E}-02(9.6 \mathrm{E}-03)$ & $\mu \mathrm{Ci} / \mathrm{mL}$ \\
\hline${ }^{246} \mathrm{Pu}$ & AEA & $1.7 \mathrm{E}-02(3.2 \mathrm{E}-03)$ & $5.1 \mathrm{E}-02(9.6 \mathrm{E}-03)$ & $\mu \mathrm{Ci} / \mathrm{mL}$ \\
\hline${ }^{241} \mathrm{Pu}$ & $\mathrm{AEA}$ & $(3.2 \mathrm{E}-03)$ & $(9.6 \mathrm{E}-03)$ & $\mu \mathrm{Ci} / \mathrm{mL}$ \\
\hline
\end{tabular}


HNF-4577, Rev. 4

Table 3. Detection Limits and Minimum Reportable Quantities for LAW Liquids (3 Sheets)

\begin{tabular}{|c|c|c|c|c|}
\hline Analyte & Method & $\begin{array}{c}\text { Estimated Quantitation } \\
\text { Limit/Minimum Detectable } \\
\text { Activity }^{(1)}\end{array}$ & $\begin{array}{c}\text { Minimum Reportable Quantity } \\
(\text { MRQ) } \\
(1)\end{array}$ & Units \\
\hline${ }^{241} \mathrm{Pu} / \mathrm{Am}$ & $\mathrm{ICP} / \mathrm{MS}$ & $1.6 \mathrm{E}+00$ & $4.8 \mathrm{E}+01$ & $\mu \mathrm{Ci} / \mathrm{mL}$ \\
\hline${ }^{242} \mathrm{Pu}$ & $\mathrm{ICP} / \mathrm{MS}$ & $1.0 \mathrm{E}-02(3.2 \mathrm{E}-03)$ & $3.0 \mathrm{E}-02(9.6 \mathrm{E}-03)$ & $\mu \mathrm{Ci} / \mathrm{mL}$ \\
\hline${ }^{241} \mathrm{Am}$ & AEA & $1.0 \mathrm{E}-02(2.4 \mathrm{E}-04)$ & $3.0 \mathrm{E}-02(7.2 \mathrm{E}-04)$ & $\mu \mathrm{Ci} / \mathrm{mL}$ \\
\hline${ }^{243} \mathrm{Am}$ & $\mathrm{ICP} / \mathrm{MS}$ & $3.2 \mathrm{E}-03$ & $9.6 \mathrm{E}-03$ & $\mu \mathrm{Ci} / \mathrm{mL}$ \\
\hline${ }^{243+244} \mathrm{Cm}$ & AEA & $5.0 \mathrm{E}-02$ & $1.5 \mathrm{E}-01$ & $\mu \mathrm{Ci} / \mathrm{mL}$ \\
\hline $\mathrm{NH}_{4} / \mathrm{NH}_{3}$ & ISE & $4.5 \mathrm{E}+01$ & $1.4 \mathrm{E}+02$ & $\mu \mathrm{g} / \mathrm{mL}$ \\
\hline $\mathrm{Cl}$ & IC & $1.0 \mathrm{E}+02$ & $3.0 \mathrm{E}+02$ & $\mu \mathrm{g} / \mathrm{mL}$ \\
\hline $\mathrm{CN}$ & distil./colorimetric & $1.5 \mathrm{E}+00$ & $4.5 \mathrm{E}+00$ & $\mu \mathrm{g} / \mathrm{mL}$ \\
\hline $\mathrm{F}$ & $\mathrm{IC}$ & $5.0 \mathrm{E}+01$ & $1.5 \mathrm{E}+02$ & $\mu \mathrm{g} / \mathrm{mL}$ \\
\hline $\mathrm{NO}_{2}$ & $\mathrm{IC}$ & $7.5 \mathrm{E}+02$ & $2.3 \mathrm{E}+03$ & $\mu \mathrm{g} / \mathrm{mL}$ \\
\hline $\mathrm{NO}_{3}$ & IC & $1.0 \mathrm{E}+03$ & $3.0 \mathrm{E}+03$ & $\mu \mathrm{g} / \mathrm{mL}$ \\
\hline $\mathrm{OH}$ & Titration & $2.5 \mathrm{E}+04$ & $7.5 \mathrm{E}+04$ & $\mu \mathrm{g} / \mathrm{mL}$ \\
\hline Oxalate & $\mathrm{IC}$ & $6.0 \mathrm{E}+02$ & $1.8 \mathrm{E}+03$ & $\mu \mathrm{g} / \mathrm{mL}$ \\
\hline $\mathrm{PO}_{4}$ & $\mathrm{IC}$ & $7.5 \mathrm{E}+02$ & $2.3 \mathrm{E}+03(2.5 \mathrm{E}+03)$ & $\mu \mathrm{g} / \mathrm{mL}$ \\
\hline $\mathrm{SO}_{4}$ & $\mathrm{IC}$ & $7.7 \mathrm{E}+02$ & $2.3 E+03$ & $\mu \mathrm{g} / \mathrm{mL}$ \\
\hline Total Alpha & prop. counter & $7.5 \mathrm{E}-02$ & $2.3 \mathrm{E}-01$ & $\mu \mathrm{Ci} / \mathrm{mL}$ \\
\hline Total Beta & beta count & TBD & TBD & $\mu \mathrm{Ci} / \mathrm{mL}$ \\
\hline $\begin{array}{l}\text { Total } \\
\text { Inorganic } \\
\text { Carbon } \\
\end{array}$ & $\begin{array}{c}\text { Persulfate/ } \\
\text { combustion furnace }\end{array}$ & $5.0 \mathrm{E}+01$ & $1.5 \mathrm{E}+02$ & $\mu \mathrm{g} / \mathrm{mL}$ \\
\hline $\begin{array}{l}\text { Total Organic } \\
\text { Carbon } \\
\end{array}$ & $\begin{array}{c}\text { Persulfate/ } \\
\text { combustion furnace }\end{array}$ & $5.0 \mathrm{E}+02$ & $1.5 \mathrm{E}+03$ & $\mu \mathrm{g} / \mathrm{mL}$ \\
\hline
\end{tabular}

Acronyms:

AEA - Alpha Energy Analysis

AMU - Atomic Mass Unit

CVAA - Cold Vapor Atomic Absorption

GEA - Gamma Energy Analysis

IC - Ion Chromatography

ICP/MS - Inductively Coupled Plasma Mass Spectroscopy

ICP/AES - Inductively Coupled Plasma Atomic Emission Spectroscopy

ISE - Ion Selective Electrode

LSC - Liquid Scintillation Counter

N/A - Not applicable

NS - Not stated

RSD - Relative Standard Deviation

$\mathrm{Wt} \%$ - Weight percent

\section{Footnotes:}

${ }^{1}$ Detection limits and MRQ's for the L\&H DQO. Where HLW or LAW DQO requirements differ from those listed, the corresponding HLW or LAW requirement is shown in parentheses. The HLW and LAW requirements apply only if testing shows that dilution is not needed to meet waste transfer requirements. If process testing determines that dilution is required for transfer, requirements for analysis of the diluted waste will be as per the test plan.

${ }^{2} \mathrm{NS}$ not stated for the L\&H DQO. In the event that testing shows that dilution is not required to meet waste transfer requirements, then the HLW and LAW MRQ's apply. If process testing determines that dilution is required for transfer, requirements for analysis of the diluted waste will be as per the test plan.

${ }^{3} \mathrm{An}$ extended counting time in the presence of high ${ }^{137} \mathrm{Cs}$ activity may be required to achieve the minimum reportable quantity for ${ }^{60} \mathrm{Co}$ and ${ }^{152} \mathrm{Eu},{ }^{154} \mathrm{Eu},{ }^{155} \mathrm{Eu}$. 
Table 4 Detection Limits and Minimum Reportable Quantities for HLW Solids (4 Sheets)

\begin{tabular}{|c|c|c|c|c|}
\hline Analyte & Method & $\begin{array}{l}\text { Estimated Quantitation } \\
\text { Limit/Minimum } \\
\text { Detectable Activity }^{(1)}\end{array}$ & $\begin{array}{l}\text { Minimum Reportable } \\
\text { Quantity (MRQ) }^{(1)}\end{array}$ & $\begin{array}{l}\text { Units (per } \\
\text { gram dried } \\
\text { solids) }\end{array}$ \\
\hline $\mathrm{Ag}$ & ICP/AES & $300(50)$ & $900(90014)$ & $\mu \mathrm{g}$ \\
\hline $\mathrm{Al}$ & $\mathrm{ICP} / \mathrm{AES}$ & $1200(110)$ & $3600(330)$ & $\mu \mathrm{g}$ \\
\hline As & ICP/MS & $20(10)$ & $60(30)$ & $\mu \mathrm{g}$ \\
\hline B & ICP/MS & $10(1)$ & $30(3)$ & $\mu \mathrm{g}$ \\
\hline $\mathrm{Ba}$ & ICP/AES & 200 & 600 & $\mu \mathrm{g}$ \\
\hline $\mathrm{Be}$ & ICP/MS & 10 & 30 & $\mu \mathrm{g}$ \\
\hline $\mathrm{Bi}$ & ICP/AES & 2000 & 6000 & $\mu \mathrm{g}$ \\
\hline $\mathrm{Ca}$ & ICP/AES & $2000(62)$ & $6000(180)$ & $\mu \mathrm{g}$ \\
\hline $\mathrm{Cd}$ & ICP/AES & $300(4)$ & $900(11)$ & $\mu \mathrm{g}$ \\
\hline $\mathrm{Ce}$ & $\mathrm{ICP} / \mathrm{MS}$ & 2 & 6 & $\mu \mathrm{g}$ \\
\hline Co & ICP/MS & $2(1)$ & $6(3)$ & $\mu \mathrm{g}$ \\
\hline $\mathrm{Cr}$ & ICP/AES & $400(40)$ & $1200(120)$ & $\mu \mathrm{g}$ \\
\hline $\mathrm{Cu}$ & ICP/AES & $200(6.5)$ & $600(18)$ & $\mu \mathrm{g}$ \\
\hline $\mathrm{F}$ & IC & 2500 & 7500 & $\mu \mathrm{g}$ \\
\hline $\mathrm{Fe}$ & ICP/AES & $400(50)$ & $1200(140)$ & $\mu \mathrm{g}$ \\
\hline $\mathrm{Hg}$ & CVAA & 0.5 & 1.5 & $\mu \mathrm{g}$ \\
\hline $\mathrm{K}$ & ICP/MS & $2000(500)$ & $6000(1500)$ & $\mu \mathrm{g}$ \\
\hline $\mathrm{La}$ & ICP/AES & $1000(20)$ & $3000(60)$ & $\mu \mathrm{g}$ \\
\hline $\mathrm{Li}$ & ICP/MS & 10 & 30 & $\mu \mathrm{g}$ \\
\hline $\mathrm{Mg}$ & ICP/AES & $1800(180)$ & $5400(540)$ & $\mu \mathrm{g}$ \\
\hline $\mathrm{Mn}$ & ICP/AES & 100 & 300 & $\mu \mathrm{g}$ \\
\hline Mo & $\mathrm{ICP} / \mathrm{MS}$ & 2 & 6 & $\mu \mathrm{g}$ \\
\hline $\mathrm{Na}$ & ICP/AES & $1800(50)$ & $5400(150)$ & $\mu \mathrm{g}$ \\
\hline $\mathrm{Nd}$ & ICP/AES & $1000(26)$ & $3000(77)$ & $\mu \mathrm{g}$ \\
\hline $\mathrm{Ni}$ & ICP/AES & $600(55)$ & $1800(160)$ & $\mu \mathrm{g}$ \\
\hline $\mathrm{P}$ & ICP/AES & $2000(200)$ & $6000(600)$ & $\mu \mathrm{g}$ \\
\hline $\mathrm{Pb}$ & ICP/AES & $1200(200)$ & $3600(600)$ & $\mu \mathrm{g}$ \\
\hline $\mathrm{Pd}$ & ICP/MS & $10(1)$ & $30(3)$ & $\mu \mathrm{g}$ \\
\hline $\operatorname{Pr}$ & ICP/MS & 2 & 6 & $\mu \mathrm{g}$ \\
\hline $\mathrm{Pu}$ & $\mathrm{ICP} / \mathrm{MS}$ & $2(8)$ & $6(24)$ & $\mu \mathrm{g}$ \\
\hline $\mathrm{Rb}$ & ICP/MS & 2 & 6 & $\mu \mathrm{g}$ \\
\hline $\mathrm{Rh}$ & ICP/MS & 2 & 6 & $\mu \mathrm{g}$ \\
\hline $\mathrm{Ru}$ & ICP/MS & $4(1)$ & $12(3)$ & $\mu \mathrm{g}$ \\
\hline$S$ & ICP/MS & $\mathrm{NS}^{2}(40)$ & $\mathrm{NS}^{2}(120)$ & $\mu \mathrm{g}$ \\
\hline $\mathrm{Sb}$ & ICP/MS & 4 & 12 & $\mu \mathrm{g}$ \\
\hline $\mathrm{Se}$ & ICP/MS & 100 & 300 & $\mu \mathrm{g}$ \\
\hline Si & ICP/AES & $10000(1000)$ & $30000(3000)$ & $\mu \mathrm{g}$ \\
\hline $\mathrm{Sr}$ & ICP/AES & 100 & 300 & $\mu \mathrm{g}$ \\
\hline $\mathrm{Ta}$ & ICP/MS & 2 & 6 & $\mu \mathrm{g}$ \\
\hline Tc & ICP/MS & (2) & (6) & $\mu \mathrm{g}$ \\
\hline
\end{tabular}


HNF-4577, Rev. 4

Table 4 Detection Limits and Minimum Reportable Quantities for HLW Solids (4 Sheets)

\begin{tabular}{|c|c|c|c|c|}
\hline Analyte & Method & \begin{tabular}{|l|} 
Estimated Quantitation \\
Limit/Minimum \\
Detectable Activity \\
\end{tabular} & $\begin{array}{l}\text { Minimum Reportable } \\
\text { Quantity (MRQ) }\end{array}$ & $\begin{array}{l}\text { Units (per } \\
\text { gram dried } \\
\text { solids) }\end{array}$ \\
\hline $\mathrm{Te}$ & ICP/MS & $6(2)$ & $18(6)$ & $\mu \mathrm{g}$ \\
\hline Th & ICP/MS & $2(200)$ & $6(600)$ & $\mu \mathrm{g}$ \\
\hline $\mathrm{Ti}$ & ICP/AES & $200(50)$ & $600(150)$ & $\mu \mathrm{g}$ \\
\hline $\mathrm{TI}$ & ICP/MS & $2(200)$ & $6(600)$ & $\mu \mathrm{g}$ \\
\hline $\mathrm{V}$ & ICP/MS & $2(0.02)$ & $6(0.06)$ & $\mu \mathrm{g}$ \\
\hline W & ICP/MS & 2 & 6 & $\mu \mathrm{g}$ \\
\hline $\mathrm{Y}$ & ICP/MS & $2(90)$ & $6(270)$ & $\mu \mathrm{g}$ \\
\hline $\mathrm{Zn}$ & ICP/AES & $400(2)$ & $1200(6)$ & $\mu \mathrm{g}$ \\
\hline $\mathrm{Zr}$ & ICP/AES & $\mathrm{NS}^{2}(200)$ & $\mathrm{NS}^{2}(600)$ & $\mu \mathrm{g}$ \\
\hline $\mathrm{Cl}$ & IC & 75 & 225 & $\mu \mathrm{g}$ \\
\hline $\mathrm{CN}^{-}$ & $\mathrm{CN}^{-}$analysis & 1 & 3 & $\mu \mathrm{g}$ \\
\hline $\mathrm{CO}_{3}^{-2}$ & $\begin{array}{l}\text { Persulfate. } \\
\text { Combustion } \\
\text { furnace }\end{array}$ & $\mathrm{NS}^{2}(10)$ & $\mathrm{NS}^{2}(30)$ & $\mu \mathrm{g}$ \\
\hline $\mathrm{NH}_{3}$ & ISE & 20 & 60 & $\mu \mathrm{g}$ \\
\hline $\mathrm{NO}_{2}{ }^{\circ}$ & IC & 150 & 450 & $\mu \mathrm{g}$ \\
\hline $\mathrm{NO}_{3}{ }^{-}$ & IC & 150 & 450 & $\mu \mathrm{g}$ \\
\hline TOC & $\begin{array}{l}\text { Persulfate. } \\
\text { Combustion } \\
\text { furnace }\end{array}$ & 20 & 60 & $\mu \mathrm{g} \mathrm{C}$ \\
\hline${ }^{3} \mathrm{H}$ & NS & NS (5.0E-03) & $\mathrm{NS}(1.5 \mathrm{E}-02)$ & $\mu \mathrm{Ci}$ \\
\hline${ }^{14} \mathrm{C}$ & $\beta-\mathrm{LSC}$ & $2.0 \mathrm{E}-04(6.0 \mathrm{E}-04)$ & $6.0 \mathrm{E}-04(1.8 \mathrm{E}-03)$ & $\mu \mathrm{Ci}$ \\
\hline${ }^{59} \mathrm{Ni}$ & $\mathrm{Sep} / \mathrm{GEA}$ & $1.0 \mathrm{E}-02$ & $3.0 \mathrm{E}-02$ & $\mu \mathrm{Ci}$ \\
\hline${ }^{60} \mathrm{Co}$ & GEA & $4.0 \mathrm{E}-02(4.0 \mathrm{E}-03)$ & $1.2 \mathrm{E}-01(1.2 \mathrm{E}-02)$ & $\mu \mathrm{Ci}$ \\
\hline${ }^{63} \mathrm{Ni}$ & $\mathrm{Sep} / \beta$-LSC & $2.0 \mathrm{E}-03$ & $6.0 \mathrm{E}-03$ & $\mu \mathrm{Ci}$ \\
\hline${ }^{90} \mathrm{Sr}^{(3)}$ & Sep/ß-GPC & $7.0 \mathrm{E}+00$ & $2.1 \mathrm{E}+01$ & $\mu \mathrm{Ci}$ \\
\hline${ }^{90} Y^{(3)}$ & Sep/B-GPC & $7.0 \mathrm{E}+00$ & $2.1 \mathrm{E}+01$ & $\mu \mathrm{Ci}$ \\
\hline${ }^{93} \mathrm{Zr}^{(8)}$ & B-LSC & $2.0 \mathrm{E}-03$ & $6.0 \mathrm{E}-03$ & $\mu \mathrm{Ci}$ \\
\hline${ }^{93} \mathrm{AMU}$ & ICP/MS & 4 & 12 & $\mu \mathrm{Ci}$ \\
\hline${ }^{93 \mathrm{~m}} \mathrm{Nb}^{(8)}$ & ICP/MS & 4 & 12 & $\mu \mathrm{Ci}$ \\
\hline${ }^{99} \mathrm{Tc}$ & ICP/MS & $\mathrm{NA}(2.0 \mathrm{E}+00)$ & NA $(6.0 \mathrm{E}+00)$ & $\mu \mathrm{Ci}$ \\
\hline${ }^{121 \mathrm{~m}} \mathrm{Sn}^{(9)}$ & Sep/GEA & $9.0 \mathrm{E}-02$ & $2.7 \mathrm{E}-01$ & $\mu \mathrm{Ci}$ \\
\hline${ }^{125} \mathrm{Sb}^{(4)}$ & GEA & $2.0 \mathrm{E}+00$ & $6.0 \mathrm{E}+00$ & $\mu \mathrm{Ci}$ \\
\hline${ }^{125 \mathrm{~m}} \mathrm{Te}^{(4)}$ & GEA & $2.0 \mathrm{E}+00$ & $6.0 \mathrm{E}+00$ & $\mu \mathrm{Ci}$ \\
\hline${ }^{126 \mathrm{~m}} \mathrm{Sb}^{(5)}$ & Sep/GEA & $6.0 \mathrm{E}-03$ & $1.8 \mathrm{E}-02$ & $\mu \mathrm{Ci}$ \\
\hline${ }^{126} \mathrm{Sb}^{(5)}$ & Sep/GEA & $6.0 \mathrm{E}-03$ & $1.8 \mathrm{E}-02$ & $\mu \mathrm{Ci}$ \\
\hline${ }^{126} \mathrm{Sn}^{(5)}$ & ICP/MS & $6.0 \mathrm{E}-03(2.0 \mathrm{E}-02)$ & $1.8 \mathrm{E}-02(6.0 \mathrm{E}-02)$ & $\mu \mathrm{Ci}$ \\
\hline${ }^{129} \mathrm{I}$ & ICP/MS & 10 & 30 & $\mu \mathrm{Ci}$ \\
\hline${ }^{135} \mathrm{Cs}$ & ICP/MS & 2 & 6 & $\mu \mathrm{Ci}$ \\
\hline
\end{tabular}


HNF-4577, Rev. 4

Table 4 Detection Limits and Minimum Reportable Quantities for HLW Solids (4 Sheets)

\begin{tabular}{|c|l|c|c|c|}
\hline Analyte & Method & $\begin{array}{l}\text { Estimated Quantitation } \\
\text { Limit/Minimum } \\
\text { Detectable Activity }\end{array}$ & $\begin{array}{l}\text { Minimum Reportable } \\
\text { Quantity (MRQ) }\end{array}$ & $\begin{array}{l}\text { Units (per } \\
\text { gram dried } \\
\text { solids) }\end{array}$ \\
\hline${ }^{137 \mathrm{~m}} \mathrm{Ba}^{(6)}$ & GEA & $3.0 \mathrm{E}-02$ & $9.0 \mathrm{E}-02$ & $\mu \mathrm{Ci}$ \\
\hline${ }^{137} \mathrm{Cs}^{(6)}$ & GEA & $3.0 \mathrm{E}-02(2.0 \mathrm{E}-02)$ & $9.0 \mathrm{E}-02(6.0 \mathrm{E}-02)$ & $\mu \mathrm{Ci}$ \\
\hline${ }^{151} \mathrm{AMU}$ & ICP/MS & 2 & 6 & $\mu \mathrm{Ci}$ \\
\hline${ }^{152} \mathrm{Eu}$ & GEA & $2.0 \mathrm{E}+00$ & $6.0 \mathrm{E}+00(2 .-\mathrm{E}+00)$ & $\mu \mathrm{Ci}$ \\
\hline${ }^{154} \mathrm{Eu}$ & GEA & $1.0 \mathrm{E}-01(2.0 \mathrm{E}-02)$ & $3.0 \mathrm{E}-01(6.0 \mathrm{E}-02)$ & $\mu \mathrm{Ci}$ \\
\hline${ }^{155} \mathrm{Eu}$ & GEA & $2.0 \mathrm{E}+00(2.0 \mathrm{E}-02)$ & $6.0 \mathrm{E}+00(6.0 \mathrm{E}-02)$ & $\mu \mathrm{Ci}$ \\
\hline${ }^{233} \mathrm{U}$ & ICP/MS & $0.2(2.0 \mathrm{E}+00)$ & $0.6(6.0 \mathrm{E}+00)$ & $\mu \mathrm{Ci}$ \\
\hline${ }^{234} \mathrm{U}$ & ICP/MS & 2 & 6 & $\mu \mathrm{Ci}$ \\
\hline${ }^{235} \mathrm{U}$ & ICP/MS & 2 & 6 & $\mu \mathrm{Ci}$ \\
\hline${ }^{236} \mathrm{U}$ & ICP/MS & 2 & 6 & $\mu \mathrm{Ci}$ \\
\hline${ }^{237} \mathrm{~Np}$ & ICP/MS & $2(6.0 \mathrm{E}-01)$ & $6(1.8 \mathrm{E}+00)$ & $\mu \mathrm{Ci}$ \\
\hline${ }^{238} \mathrm{Pu}$ & Sep/AEA & $2.0 \mathrm{E}-02(2.0 \mathrm{E}-05)$ & $6.0 \mathrm{E}-02(6.0 \mathrm{E}-05)$ & $\mu \mathrm{Ci}$ \\
\hline${ }^{238} \mathrm{U}$ & ICP/MS & 2 & 6 & $\mu \mathrm{Ci}$ \\
\hline${ }^{239} \mathrm{Pu}{ }^{(7)}$ & Sep/AEA & $2.0 \mathrm{E}-02(2.0 \mathrm{E}+00)$ & $6.0 \mathrm{E}-02(6.0 \mathrm{E}+00)$ & $\mu \mathrm{Ci}$ \\
\hline${ }^{240} \mathrm{Pu}{ }^{(7)}$ & Sep/AEA & $2.0 \mathrm{E}-02$ & $6.0 \mathrm{E}-02$ & $\mu \mathrm{Ci}$ \\
\hline${ }^{241} \mathrm{Am}$ & Sep/AEA & $6.0 \mathrm{E}-03(4.0 \mathrm{E}-04)$ & $1.8 \mathrm{E}-02(1.2 \mathrm{E}-03)$ & $\mu \mathrm{Ci}$ \\
\hline${ }^{241} \mathrm{AMU}$ & ICP/MS & 2 & 6 & $\mu \mathrm{Ci}$ \\
\hline${ }^{242} \mathrm{Cm}{ }^{(10)}$ & Sep/AEA & $4.0 \mathrm{E}-03$ & $1.2 \mathrm{E}-02$ & $\mu \mathrm{Ci}$ \\
\hline${ }^{242 \mathrm{~m}} \mathrm{Am}{ }^{(10)}$ & Sep/AEA & $4.0 \mathrm{E}-03$ & $1.2 \mathrm{E}-02$ & $\mu \mathrm{Ci}$ \\
\hline${ }^{242} \mathrm{Pu}$ & ICP/MS & 0.2 & 0.6 & $\mu \mathrm{Ci}$ \\
\hline${ }^{242} \mathrm{Pu}$ & Sep/AEA & $2.0 \mathrm{E}-02$ & $6.0 \mathrm{E}-02$ & $\mu \mathrm{Ci}$ \\
\hline${ }^{243} \mathrm{Am}$ & ICP/MS & 2 & 6 & $\mu \mathrm{Ci}$ \\
\hline${ }^{243+244} \mathrm{Cm}$ & Sep/AEA & $4.0 \mathrm{E}-03(2.0 \mathrm{E}-05)$ & $1.2 \mathrm{E}-02(6.0 \mathrm{E}-05)$ & $\mu \mathrm{Ci}$ \\
\hline & & & & \\
\hline
\end{tabular}




\section{HNF-4577, Rev. 4}

Acronyms:

AEA - Alpha Energy Analysis

GEA - Gamma Energy Analysis

GPC - Gas flow proportional counter

ICP/MS - Inductively Coupled Plasma Mass Spectroscopy

ICP/AES - Inductively Coupled Plasma Atomic Emission Spectroscopy

LSC - Liquid Scintillation Counter

NA - Not applicable

NM - Not measured

Sep - separation required

TIMS - Thermal Ionization Mass Spectrometry

Notes:

'Detection limits and MRQ's for the L\&H DQO. Where HLW or LAW DQO requirements differ from those listed, the corresponding HLW or HAW requirement is shown in parentheses. The HLW and LAW requirements apply only if testing shows that dilution is not needed to meet waste transfer requirements. If process testing determines that dilution is required for transfer, requirements for analysis of the diluted waste will be as per the test plan.

${ }^{2} \mathrm{NS}$ not stated for the L\&H DQO. In the event that testing shows that dilution is not required to meet waste transfer requirements, then the HLW and LAW MRQ's apply. If process testing determines that dilution is required for transfer, requirements for analysis of the diluted waste will be as per the test plan.

${ }^{3}$ Combined analysis of ${ }^{90} \mathrm{Sr}$ and ${ }^{90} \mathrm{Y}$

${ }^{4}$ Combined analysis of ${ }^{125} \mathrm{Sb}$ and ${ }^{125 \mathrm{~m}} \mathrm{Te}$

${ }^{5}$ Combined analysis of ${ }^{126} \mathrm{Sn},{ }^{126 \mathrm{~m}} \mathrm{Sb}$, and ${ }^{126} \mathrm{Sb}$

${ }^{6} \mathrm{Combined}$ analysis of ${ }^{137} \mathrm{Cs}$ and ${ }^{137 \mathrm{~m}} \mathrm{Ba}$

${ }^{7}$ Combined analysis of ${ }^{239} \mathrm{Pu}$ and ${ }^{240} \mathrm{Pu}$.

${ }^{8}$ Combined analysis of ${ }^{93 \mathrm{~m}} \mathrm{Nb}$ and ${ }^{93} \mathrm{Zr}$

${ }^{9}$ No method currently available

${ }^{10} \mathrm{Combined}$ analysis of ${ }^{242} \mathrm{Am},{ }^{242 \mathrm{~m}} \mathrm{Am}$, and ${ }^{242} \mathrm{Cm}$ 
HNF-4577, Rev. 4

\subsection{QUALITY ASSURANCE AND QUALITY CONTROL}

Processes, services, activities, and conditions adverse to quality which do not conform to requirements specified in this SAP or references herein shall be controlled to prevent inadvertent use. Nonconforming sampling and analysis processes shall be identified, controlled, reported, and dispositioned as required by the Nonconformance Item Reporting and Control (CHG 1999).

\subsection{LABORATORY OPERATIONS}

Laboratories performing analyses in support of this SAP shall have approved and implemented Quality Assurance (QA) Plans. These QA plans shall meet the Hanford Analytical Services Quality Assurance Requirements Document (DOE-RL 1998) minimum requirements as the baseline for laboratory quality systems. The 222-S Laboratory Quality Assurance Plan (Markel 1999) specifies the requirements for assuring the quality of sample analysis conducted at the 222-S Laboratory. Quality requirements for conducting Characterization Project sampling and analysis are described in Tank Waste Remediation System Characterization Project, Quality Policies (Board 1998) and this SAP. Characterization Project sampling and analysis shall be conducted in conformance with these requirements.

Analytical quality control (QC) requirements (duplicates, spikes, blanks, laboratory control samples) are identified in Tables 1,2,5, and 6. The laboratory shall also use calibration and calibration check standards appropriate for the analytical instrumentation being used (see DOERL [1998] for definitions of QC samples and standards). The criteria presented are goals for demonstrating reliable method performance. It is understood that the laboratory will follow its internal QC system for required actions whenever QC failures occur. If sample QC failures occur, or if all analyses cannot be performed (e.g., insufficient sample), analysts shall consult with supervisors/customers to determine the proper action. The laboratory should provide a suggested course of action at that time. All sample QC failures and limitations on the associated data shall be discussed in the narrative of the data report. Proper notification of all data not meeting QC requirements shall be included with the data. It should be noted that the L\&H DQO requires specific quality control/quality assurance requirements. These requirements are outlined in section 7.7 of the L\&H DQO.

\subsection{SAMPLE COLLECTION}

Before sampling can be performed on a tank, available risers must be identified for use in the sampling event. The selected risers must be inspected and prepared to confirm their ability to be used in sampling. Safety hazards must be identified and special precautions must be taken if needed. If deemed necessary by the sampling cognizant engineers and tank coordinator, video surveillance should be performed to identify any potential problems that may occur during the sampling event.

Samples are to be taken from a tank and shipped to the performing laboratory by CPO in accordance with the respective work packages. The chain-of-custody forms for these work packages shall identify samples by a unique number and state the type of sampler used (retained gas sampler or universal sampler) for each sample before being shipped to the 222-S Laboratory. Approved procedure TO-080-090 [Load/Transport Sample Cask(s)] is to be used during the 


\section{HNF-4577, Rev. 4}

sampling event. Pertinent sampling information (e.g., unusual waste characteristics, X-ray scan results, $\mathrm{LiBr}$ solution used, or detecting debris) should be noted in the comment section of the chain-of-custody form.

Characterization Project Operations should transport each sample collected to the performing laboratory within three calendar days of removing the sample from the tank. A verbal notification by CPO is to be made to the 222-S Laboratory at 373-2435 at least 24 hours in advance of an expected shipment. 
HNF-4577, Rev. 4

Table 5. Quality Control Parameters for Liquid Analysis (3 Sheets)

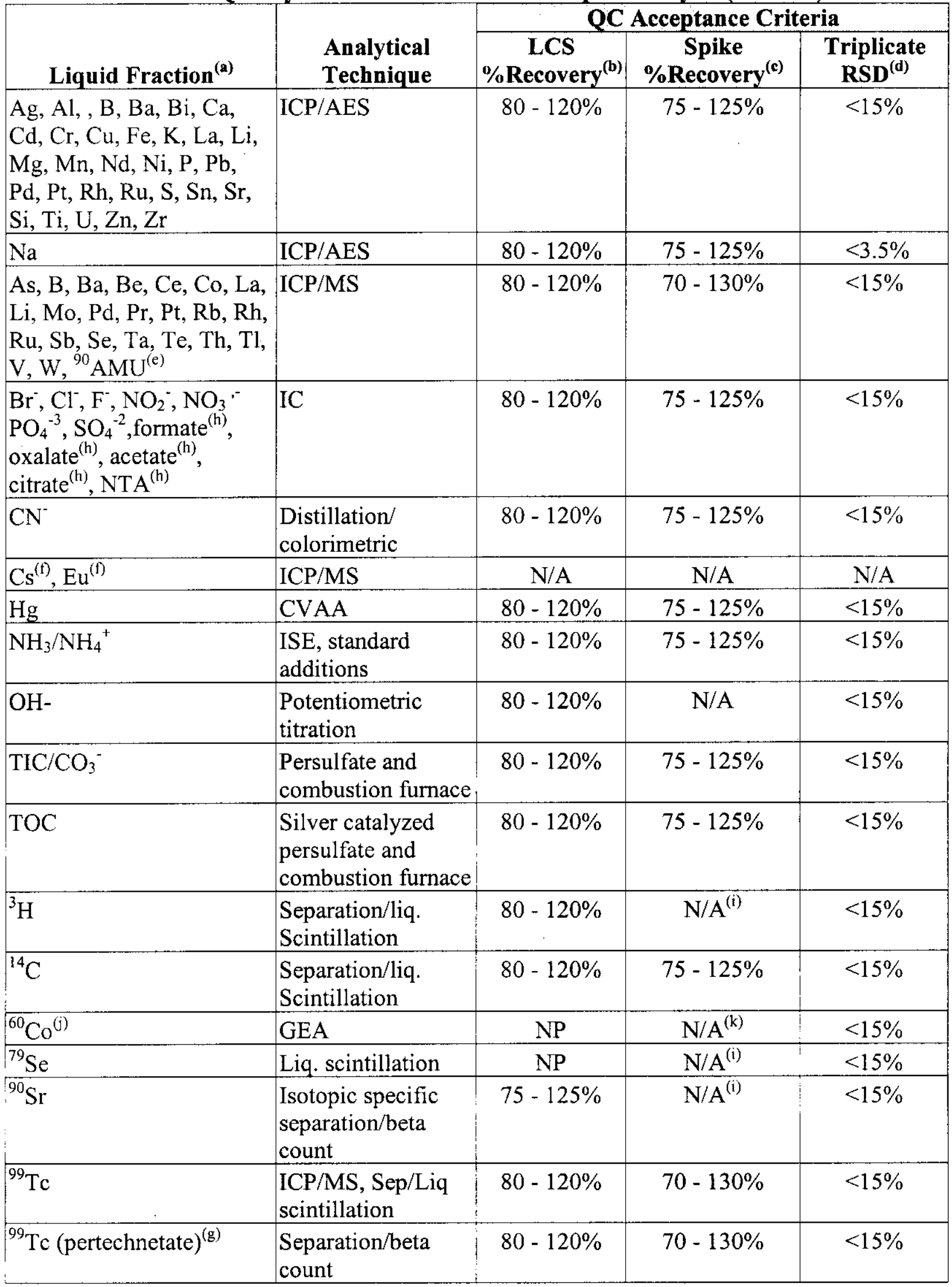




\section{HNF-4577, Rev. 4}

Table 5. Quality Control Parameters for Liquid Analysis (3 Sheets)

\begin{tabular}{|c|c|c|c|c|}
\hline \multirow[b]{2}{*}{ Liquid Fraction $^{(a)}$} & \multirow[b]{2}{*}{$\begin{array}{l}\text { Analytical } \\
\text { Technique }\end{array}$} & \multicolumn{3}{|c|}{ QC Acceptance Criteria } \\
\hline & & $\begin{array}{c}\text { LCS } \\
\text { \% Recovery }\end{array}$ & $\begin{array}{c}\text { Spike } \\
\text { \%Recovery } \\
\text { \% }\end{array}$ & $\begin{array}{c}\text { Triplicate } \\
\text { RSD }^{(d)}\end{array}$ \\
\hline${ }^{125} \mathrm{Sb}$ & GEA & TBD & TBD & TBD \\
\hline${ }^{126} \mathrm{Sn}$ & ICP/MS & $80-120 \%$ & $70-130 \%$ & $<15 \%$ \\
\hline${ }^{129} \mathrm{I}$ & ICP/MS & $80-120 \%$ & $70-130 \%$ & $<15 \%$ \\
\hline${ }^{129} \mathrm{I}$ & Separation/GEA & $\mathrm{NP}$ & $\mathrm{N} / \mathrm{A}^{(\mathrm{k})}$ & $<15 \%$ \\
\hline${ }^{137} \mathrm{Cs}$ & GEA & NP & $\mathrm{N} / \mathrm{A}^{(\mathrm{k})}$ & $<15 \%$ \\
\hline${ }^{152} \mathrm{Eu}^{(\mathrm{i})}$ & GEA & NP & $\mathrm{N} / \mathrm{A}^{(\mathrm{k})}$ & $<15 \%$ \\
\hline${ }^{154} \mathrm{Eu}^{(\mathrm{i}, \mathrm{j})}$ & GEA & $\mathrm{NP}$ & $N / A^{(k)}$ & $<15 \%$ \\
\hline${ }^{155} \mathrm{Eu}^{(\mathrm{i}, \mathrm{j}\}}$ & GEA & $\mathrm{NP}$ & $\mathrm{N} / \mathrm{A}^{(\mathrm{k})}$ & $<15 \%$ \\
\hline${ }^{231} \mathrm{~Pa}$ & ICP/MS & TBD & TBD & $\mathrm{TBD}$ \\
\hline${ }^{233} \mathrm{U}$ & ICP/MS & $90-110 \%$ & $75-125 \%$ & $<15 \%$ \\
\hline${ }^{234} \mathrm{U}$ & ICP/MS & $90-110 \%$ & $75-125 \%$ & $<15 \%$ \\
\hline${ }^{235} \mathrm{U}$ & ICP/MS & $90-110 \%$ & $75-125 \%$ & $<15 \%$ \\
\hline${ }^{236} \mathrm{U}$ & ICP/MS & $90-110 \%$ & $75-125 \%$ & $<15 \%$ \\
\hline${ }^{238} \mathrm{U}$ & ICP/MS & $80-120 \%$ & $70-130 \%$ & $<15 \%$ \\
\hline${ }^{237} \mathrm{~Np}^{(1)}$ & ICP/MS & $90-110 \%$ & $75-125 \%$ & $<15 \%$ \\
\hline Total $\mathrm{Pu}$ & Sum of Isotopes & $\mathrm{N} / \mathrm{A}$ & N/A & N/A \\
\hline${ }^{238} \mathrm{Pu},{ }^{239} \mathrm{Pu},{ }^{240} \mathrm{Pu}^{(1)}$ & Separation/AEA & NP & $N / A^{(i)}$ & $<15 \%$ \\
\hline${ }^{241} \mathrm{Pu} / \mathrm{Am},{ }^{242} \mathrm{Pu}$ & ICP/MS & $80-120 \%$ & $70-130 \%$ & $<15 \%$ \\
\hline${ }^{241} \mathrm{Am}$ & Separation/AEA & NP & $\mathrm{N} / \mathrm{A}^{(\mathrm{i})}$ & $<15 \%$ \\
\hline${ }^{242} \mathrm{Cm}$ & Separation/AEA & NP & $\mathrm{N} / \mathrm{A}^{(\mathrm{i})}$ & $<15 \%$ \\
\hline${ }^{243} \mathrm{Am} / \mathrm{Cm}$ & ICP/MS & $90-110 \%$ & $75-125 \%$ & $<15 \%$ \\
\hline${ }^{243+244} \mathrm{Cm}$ & Separation/AEA & NP & $N / A^{(i)}$ & $<15 \%$ \\
\hline Total Alpha ${ }^{(l)}$ & $\begin{array}{l}\text { Proportional } \\
\text { counter }\end{array}$ & $70-130 \%$ & $\begin{array}{c}75-125 \% \\
\text { (80-120\% RPD } \\
\text { for Safety } \\
\text { Screening }\end{array}$ & $<15 \%{ }^{(0)}$ \\
\hline Total Beta & Beta counting & $70-130 \%$ & $70-130 \%$ & $<15 \%$ \\
\hline Total Gamma & $\begin{array}{l}\text { GEA-Sum of } \\
\text { isotopes }\end{array}$ & N/A & N/A & N/A \\
\hline Specific Gravity & Density & N/A & N/A & N/A \\
\hline $\mathrm{Wt}_{\mathrm{t}} \%$ dissolved solids $^{(\mathrm{m})}$ & Gravimetric & $80-120 \%$ & N/A & $<21 \%$ \\
\hline
\end{tabular}

Acronyms:

AEA - Alpha Energy Analysis

CVAA - Cold Vapor Atomic Absorption

GEA - Gamma Energy Analysis

IC - Ion Chromatography

ICP/MS - Inductively Coupled Plasma Mass Spectroscopy

ICP/AES - Inductively Coupled Plasma Atomic Emission Spectroscopy

LCS - Laboratory Control Standard 


\section{HNF-4577, Rev. 4}

N/A - Not applicable

NP - not performed

RPD - relative percent difference

TBD - To be determined

RSD - Relative Standard Deviation

Wt \% - Weight Percent

\section{Footnotes:}

(a) Analytes for the Solubility Screening Test are a subset of this analyte list.

(b) LCS $=$ Laboratory Control Standard. This standard is carried through the entire method. The accuracy of a method is usually expressed as the percent recovery of the LCS. The LCS is a matrix with known concentration of analytes processed with each preparation and analyses batch. It is expressed as percent recovery; i.e., the amount measured, divided by the known concentration, times 100 .

(c) For some methods, the sample accuracy is expressed as the percent recovery of a matrix spike sample. It is expressed as percent recovery; i.e., the amount measured, less the amount in the sample, divided by the spike added, times 100 . One matrix spike is performed per analytical batch. Samples are batched with similar matrices.

For other analytes, the accuracy is determined based on use of serial dilutions.

(d) $\mathrm{RSD}=$ Relative Standard Deviation between the samples, defined as: (standard deviation of the mean/mean) $\times 100$. Sample precision is estimated by analyzing replicates taken separately through preparation and analysis. Acceptable sample precision is usually $<15 \%$ RSD if the sample result is at least 10 times the instrument detection limit.

(e) ICP-MS mass unit 90 includes ${ }^{90} \mathrm{Sr},{ }^{90} \mathrm{Y}$, and ${ }^{90} \mathrm{Zr}$.

(f) Total Cs and Eu are sums of all isotopes; therefore, spiking and LCS do not apply.

(g) The radiochemical analysis for pertechnetate is to be performed on an unoxidized sample. That is, do NOT use a sample preparation that converts all Tc to the pertechnetate form.

(h) Secondary analytes for the L\&H DQO

(i) Matrix spike analyses are not required for this method because a tracer is used to correct for analyte loss during sample preparation and analysis. The result generated using the tracer accounts for any inaccuracy of the method on the matrix. The reported results reflect this correction.

(j) An extended counting time in the presence of high ${ }^{137} \mathrm{Cs}$ activity may be required to achieve the minimum reportable quantity for ${ }^{60} \mathrm{Co}$ and ${ }^{154} \mathrm{Eu},{ }^{155} \mathrm{Eu}$.

(k) The measurement is a direct reading of the energy and the analysis is not affected by the sample matrix; therefore, a matrix spike is not required.

(l) The sum of ${ }^{238} \mathrm{Pu},{ }^{239} \mathrm{Pu},{ }^{240} \mathrm{Pu}$, and ${ }^{241} \mathrm{Am}$ activities will be used as a measurement of alpha-emitting TRU when total alpha measurement in the liquid fraction is equal to or exceeds $6.0 \mathrm{E}-05 \mathrm{Ci} / \mathrm{L}$ for Envelopes $\mathrm{A}$ and $\mathrm{B}$ and $4.0 \mathrm{E}-04 \mathrm{Ci} / \mathrm{L}$ for Envelope $\mathrm{C}$. The selected trigger values correspond to $70 \%$ of the $\mathrm{LAW}$ envelope limits for TRU. The selected isotopes account for greater than $95 \%$ of the alpha-emitting TRU activity based on previous analysis of Phase I candidate tank waste (Esch 1997a, 1997b, 1997c). Additional isotopes which are defined as alpha-emitting TRU (e.g., ${ }^{237} \mathrm{~Np},{ }^{242} \mathrm{Pu},{ }^{242} \mathrm{Cm},{ }^{243} \mathrm{Am}$, and ${ }^{243+244} \mathrm{Cm}$ ) are not used to calculate total TRU activity because the MDAs for these isotopes are large in comparison with the envelope limits and it is expected that their concentrations are well below the minimal detectable activities (MDA). Note that ${ }^{241} \mathrm{Pu}$ is a beta-emitting TRU whose analysis, along with ${ }^{242} \mathrm{Cm}$, is required specifically for class $\mathrm{C}$ waste determination.

(m) Weight percent dissolved solids method is described in L\&H DQO Section 7.3.4. 
HNF-4577, Rev. 4

Table 6. Quality Control Parameters for Solids Analysis (3 Sheets)

\begin{tabular}{|c|c|c|c|c|}
\hline \multirow[b]{2}{*}{ Solids Fraction ${ }^{(a)}$} & \multirow[b]{2}{*}{$\begin{array}{l}\text { Analytical } \\
\text { Technique }\end{array}$} & \multicolumn{3}{|c|}{ QC Acceptance Criteria } \\
\hline & & $\begin{array}{c}\text { LCS \% } \\
\text { Recovery }^{\left({ }^{b}\right)}\end{array}$ & $\begin{array}{r}\text { Spike \% } \\
\text { Recovery }\end{array}$ & $\begin{array}{c}\text { Triplicate } \\
\text { RSD }^{(\mathbf{d})}\end{array}$ \\
\hline $\begin{array}{l}\mathrm{Ag}, \mathrm{Al}, \mathrm{B}, \mathrm{Ba}, \mathrm{Bi}, \mathrm{Ca} \\
\mathrm{Cd}, \mathrm{Cr}, \mathrm{Cu}, \mathrm{Fe}, \mathrm{K}, \mathrm{La} \\
\mathrm{Li}, \mathrm{Mg}, \mathrm{Mn}, \mathrm{Nd}, \mathrm{Ni}, \mathrm{P} \\
\mathrm{Pb}, \mathrm{S}, \mathrm{Si}, \mathrm{Sr}, \mathrm{Tc}, \mathrm{Ti}, \mathrm{U} \\
\mathrm{Zn}, \mathrm{Zr}, \mathrm{Y}\end{array}$ & ICP/AES & $80-120 \%$ & $75-125 \%$ & $<15 \%$ \\
\hline $\mathrm{Na}$ & ICP/AES & $80-120 \%$ & $75-125 \%$ & $<3.5 \%$ \\
\hline $\begin{array}{l}\text { As, B, Be, Ce, Co, K, } \\
\text { Li, Mo, Pd, Pr, Rb, Rh, } \\
\text { Ru, Sb, Se, Ta, Te, Th, } \\
\text { Tl, V, W, }{ }^{90} \mathrm{AMU}^{(\mathbf{e})}\end{array}$ & ICP/MS & $80-120 \%$ & $70-130 \%$ & $<15 \%$ \\
\hline $\begin{array}{l}\text { Acetate, } \mathrm{Br}, \mathrm{Cl} \text {, citrate, } \\
\mathrm{F}^{-} \text {, formate, } \mathrm{NO}_{2}^{-}, \\
\mathrm{NO}_{3}^{-} \text {, oxalatc, } \mathrm{NTA}, \\
\mathrm{PO}_{4}, \mathrm{SO}_{4}\end{array}$ & IC & $80-120 \%$ & $75-125 \%$ & $<15 \%$ \\
\hline $\mathrm{CN}^{-}$ & $\begin{array}{l}\text { Distillation/ } \\
\text { colorimetric }\end{array}$ & $80-120 \%$ & $75-125 \%$ & $<15 \%$ \\
\hline $\mathrm{Cs}^{(1)}$ & ICP/MS & N/A & N/A & $\mathrm{N} / \mathrm{A}$ \\
\hline $\mathrm{Hg}$ & CVAA & $80-120 \%$ & $75-125 \%$ & $<15 \%$ \\
\hline $\mathrm{NH}_{3} / \mathrm{NH}_{4}{ }^{+}$ & $\begin{array}{l}\text { ISE, standard } \\
\text { additions }\end{array}$ & $80-120 \%$ & $75-125 \%$ & $<15 \%$ \\
\hline $\mathrm{TIC} / \mathrm{CO}_{3}^{-}$ & $\begin{array}{l}\text { Persulfate and } \\
\text { combustion furnace }\end{array}$ & $80-120 \%$ & $75-125 \%$ & $<15 \%$ \\
\hline TOC & $\begin{array}{l}\text { silver catalyzed } \\
\text { persulfate and } \\
\text { combustion furnace }\end{array}$ & $80-120 \%$ & $75-125 \%$ & $<15 \%$ \\
\hline$Y$ & $\begin{array}{l}\text { Derived from } \\
\text { calculation }\end{array}$ & $\mathrm{N} / \mathrm{A}$ & N/A & N/A \\
\hline${ }^{3} \mathrm{H}$ & Sep/LSC & $80-120 \%$ & $\mathrm{~N} / \mathrm{A}^{(\mathrm{g})}$ & $<15 \%$ \\
\hline${ }^{14} \mathrm{C}$ & $\mathrm{Sep} / \mathrm{LSC}$ & $80-120 \%$ & $75-125 \%$ & $<15 \%$ \\
\hline${ }^{63} \mathrm{Ni}^{\text {hh }}$ & ICP/MS & $80-120 \%$ & $70-130 \%$ & $<15 \%$ \\
\hline${ }^{59} \mathrm{Ni}^{(h)}$ & ICP/MS & $80-120 \%$ & $70-130 \%$ & $<15 \%$ \\
\hline${ }^{59} \mathrm{Ni}^{(h)}$ & Sep/GEA & $\mathrm{NP}$ & $\mathrm{N} / \mathrm{A}^{(1)}$ & $<15 \%$ \\
\hline${ }^{63} \mathrm{Ni}^{(\mathrm{h})}$ & $\begin{array}{l}\text { Isotopic specific } \\
\text { separation/beta-LSC }\end{array}$ & NP & $\mathrm{N} / \mathrm{A}^{(\mathrm{g})}$ & $<15 \%$ \\
\hline${ }^{60} \mathrm{Co}^{(j)}$ & GEA & $80-120 \%$ & $\mathrm{~N} / \mathrm{A}^{(\mathrm{l})}$ & $<15 \%$ \\
\hline${ }^{90} \mathrm{Sr}^{(\mathrm{k})}$ & $\begin{array}{l}\text { Isotopic specific } \\
\text { separation/beta } \\
\text { count }\end{array}$ & $75-125 \%$ & $N / A^{(g)}$ & $<15 \%$ \\
\hline${ }^{90} \mathrm{Y}^{(\mathrm{k}),(\mathrm{h})}$ & $\begin{array}{l}\text { Isotopic specific } \\
\text { separation/beta } \\
\text { count }\end{array}$ & $75-125 \%$ & $\mathrm{~N} / \mathrm{A}^{(\mathrm{g})}$ & $<15 \%$ \\
\hline${ }^{93} \mathrm{Zr}^{(h),(1)}$ & Beta-LSC & NP & $N / A^{(g)}$ & $<15 \%$ \\
\hline
\end{tabular}


HNF-4577, Rev. 4

Table 6. Quality Control Parameters for Solids Analysis (3 Sheets)

\begin{tabular}{|c|c|c|c|c|}
\hline \multirow[b]{2}{*}{ Solids Fraction $^{(a)}$} & \multirow[b]{2}{*}{$\begin{array}{l}\text { Analytical } \\
\text { Technique }\end{array}$} & \multicolumn{3}{|c|}{ QC Acceptance Criteria } \\
\hline & & $\begin{array}{c}\text { LCS \% } \\
\text { Recovery }\end{array}$ & $\begin{array}{c}\text { Spike \% } \\
\text { Recovery }\end{array}$ & $\begin{array}{c}\text { Triplicate } \\
\text { RSD }^{(\mathbf{d})}\end{array}$ \\
\hline${ }^{93} \mathrm{AMU}$ & ICP/MS & $80-120 \%$ & $70-130 \%$ & $<15 \%$ \\
\hline${ }^{99} \mathrm{Tc}$ & ICP/MS, Sep/LSC & $80-120 \%$ & $70-130 \%$ & $<15 \%$ \\
\hline${ }^{121 \mathrm{~m}} \mathrm{Sn}^{(\mathrm{h})}$ & Sep/GEA & $\mathrm{NP}$ & $\mathrm{N} / \mathrm{A}^{(1)}$ & $<15 \%$ \\
\hline $125 \mathrm{Sb}^{(m)}$ & GEA & $\mathrm{NP}$ & $\mathrm{N} / \mathrm{A}^{(\mathrm{l})}$ & $<15 \%$ \\
\hline${ }^{126} \mathrm{Sb}^{(\mathrm{n}),(\mathrm{h})}$ & Sep/GEA & NP & $\mathrm{N} / \mathrm{A}^{(\mathrm{I})}$ & $<15 \%$ \\
\hline${ }^{126 m} \mathrm{Sb}^{(\mathrm{h}),(\mathrm{n})}$ & Sep/GEA & $\mathrm{NP}$ & $\mathrm{N} / \mathrm{A}^{(1)}$ & $<15 \%$ \\
\hline${ }^{126} \mathrm{Sn}^{(\mathrm{n})}$ & ICP/MS & $80-120 \%$ & $70-130 \%$ & $<15 \%$ \\
\hline${ }^{129} \mathrm{I}$ & $\mathrm{Sep} / \mathrm{GEA}$ & $\mathrm{NP}$ & $\mathrm{N} / \mathrm{A}^{(1)}$ & $<15 \%$ \\
\hline${ }^{135} \mathrm{Cs}^{(\mathrm{h})}$ & ICP/MS & $80-120 \%$ & $70-130 \%$ & $<15 \%$ \\
\hline${ }^{137} \mathrm{Cs}$ & GEA & $\mathrm{NP}$ & $\mathrm{N} / \mathrm{A}^{(1)}$ & $<15 \%$ \\
\hline${ }^{151} \mathrm{Sm}$ & \begin{tabular}{|l|} 
Isotopic specific \\
separation/beta-LSC
\end{tabular} & $\mathrm{NP}$ & $\mathrm{N} / \mathrm{A}^{(\mathrm{g})}$ & $<15 \%$ \\
\hline${ }^{152} \mathrm{Eu}^{(\mathrm{j})}$ & GEA & NP & $\mathrm{N} / \mathrm{A}^{(1)}$ & $<15 \%$ \\
\hline${ }^{154} \mathrm{Eu}^{(1)}$ & GEA & NP & $\mathrm{N} / \mathrm{A}^{(1)}$ & $<15 \%$ \\
\hline${ }^{155} \mathrm{Eu}^{(i)}$ & GEA & NP & $\mathrm{N} / \mathrm{A}^{(1)}$ & $<15 \%$ \\
\hline${ }^{233} \mathrm{U}$ & ICP/MS & $90-110 \%$ & $75-125 \%$ & $<15 \%$ \\
\hline${ }^{234} \mathrm{U}$ & ICP/MS & $90-110 \%$ & $75-125 \%$ & $<15 \%$ \\
\hline${ }^{235} \mathrm{U}$ & $\mathrm{ICP} / \mathrm{MS}$ & $90-110 \%$ & $75-125 \%$ & $<15 \%$ \\
\hline${ }^{236} \mathrm{U}$ & ICP/MS & $90-110 \%$ & $75-125 \%$ & $<15 \%$ \\
\hline${ }^{238} \mathrm{U}$ & ICP/MS & $80-120 \%$ & $70-130 \%$ & $<15 \%$ \\
\hline${ }^{237} \mathrm{~Np}^{(0)}$ & ICP/MS & $90-110 \%$ & $75-125 \%$ & $<15 \%$ \\
\hline Total Pu & Sum of Isotopes & N/A & N/A & N/A \\
\hline${ }^{238} \mathrm{Pu},{ }^{239} \mathrm{Pu},{ }^{240} \mathrm{Pu}{ }^{(0)}$ & Sep/AEA & NP & $70-130 \%$ & $<15 \%$ \\
\hline${ }^{241} \mathrm{Pu} / \mathrm{Am},{ }^{242} \mathrm{Pu}^{(0)}$ & ICP/MS & $80-120 \%$ & $70-130 \%$ & $<15 \%$ \\
\hline${ }^{241} \mathrm{Am}^{(0)}$ & Sep/AEA & $80-120 \%$ & $\mathrm{~N} / \mathrm{A}^{(\mathrm{g})}$ & $<15 \%$ \\
\hline${ }^{242} \mathrm{Cm}^{(0)}$ & Sep/AEA & $\overline{\mathrm{NP}}$ & $\mathrm{N} / \mathrm{A}^{(\mathrm{g})}$ & $<15 \%$ \\
\hline${ }^{243} \mathrm{Am} / \mathrm{Cm}^{(0)}$ & $\mathrm{ICP} / \mathrm{MS}$ & $90-110 \%$ & $75-125 \%$ & $<15 \%$ \\
\hline $243+244 \mathrm{Cm}^{(0)}$ & Sep/AEA & NP & $\mathrm{N} / \mathrm{A}^{(\mathrm{g})}$ & $<15 \%$ \\
\hline Total Alpha ${ }^{(0)}$ & Proportional counter & $70-130 \%$ & $75-125 \%$ & $<15 \%$ \\
\hline Total Beta & Beta counting & $70-130 \%$ & $70-130 \%$ & $<15 \%$ \\
\hline Total Gamma & $\begin{array}{l}\text { GEA-Sum of } \\
\text { isotopes }\end{array}$ & N/A & N/A & N/A \\
\hline Bulk density & Gravimetric & N/A & N/A & N/A \\
\hline $\mathrm{Wt} \%$ solids $^{(p)}$ & gravimetric & $80-120 \%$ & N/A & $<21 \%$ \\
\hline Wt $\%$ oxide ${ }^{(p)}$ & gravimetric & TBD & TBD & TBD \\
\hline Shear Strength & Rot Viscometer & N/A & N/A & N/A \\
\hline Particle size & $\begin{array}{l}\text { Particle Size } \\
\text { Analysis }\end{array}$ & N/A & N/A & N/A \\
\hline
\end{tabular}

Acronyms:

AEA - Alpha Energy Analysis

CVAA - Cold Vapor Atomic Absorption

GEA - Gamma Energy Analysis 


\section{HNF-4577, Rev. 4}

IC - Ion Chromatography

ICP/MS - Inductively Coupled Plasma Mass Spectroscopy

ICP/AES - Inductively Coupled Plasma Atomic Emission Spectroscopy

LCS - Laboratory Control Standard

N/A - Not applicable

TBD - To be determined

RSD - Relative Standard Deviation

$\mathrm{Wt} \%$ - Weight Percent

NP - Not performed

N/A - Not available

\section{Footnotes:}

(a) Analytes for the Solubility Screening Test are a subset of this analyte list

(b) LCS = Laboratory Control Standard. This standard is carried through the entire method. The accuracy of a method is usually expressed as the percent recovery of the LCS. The LCS is a matrix with known concentration of analytes processed with each preparation and analyses batch. It is expressed as percent recovery; i.e., the amount measured, divided by the known concentration, times 100 .

(c) For some methods, the sample accuracy is expressed as the percent recovery of a matrix spike sample. It is expressed as percent recovery; i.e., the amount measured, less the amount in the sample, divided by the spike added, times 100 . One matrix spike is performed per analytical batch. Samples are batched with similar matrices. For other analytes, the accuracy is determined based on use of serial dilutions as described in Section 7.6.2.2.

(d) $\mathrm{RSD}=$ Relative Standard Deviation between the samples. Sample precision is estimated by analyzing replicates taken separately through preparation and analysis. Acceptable sample precision is usually $<15 \%$ RSD if the sample result is at least 10 times the instrument detection limit.

$\mathrm{RSD}=($ standard deviation of the mean/mean) $\times 100$

(e) ICP/MS mass unit 90 includes ${ }^{90} \mathrm{Sr},{ }^{90} \mathrm{Y}$, and ${ }^{90} \mathrm{Zr}$.

(f) Total Cs and Eu are sums of all isotopes; therefore, spiking and LCS do not apply.

(g) Matrix spike analyses are not required for this method because a tracer is used to correct for analyte loss during sample preparation and analysis. The result is generated using the tracer accounts for an inaccuracy of the method on the matrix. The reported results reflect this correction.

(h) Radionuclide only required for Waste Acceptance Product Specifications (WAPS) justification. Analysis is lower priority if unique separation or analysis is required.

(i) The measurement is a direct reading of the energy and the analysis is not affected by the sample matrix; therefore, a matrix spike is not required.

(j) An extended counting time in the presence of relatively high gamma-activity may be required to achieve the minimum reportable quantity for ${ }^{60} \mathrm{Co}$ and ${ }^{154} \mathrm{Eu},{ }^{155} \mathrm{Eu}$.

(k) Combined analysis of ${ }^{90} \mathrm{Sr}$ and ${ }^{90} \mathrm{Y}$.

(l) Combined analysis with ${ }^{93 \mathrm{~m}} \mathrm{Nb}$.

(m) Combined analysis with ${ }^{125 \mathrm{~m}} \mathrm{Te}$.

(n) Combined analysis of ${ }^{126} \mathrm{Sn},{ }^{126} \mathrm{Sb}$, and ${ }^{126 \mathrm{~m}} \mathrm{Sb}$.

(o) Trigger level based on total alpha and specific isotopes to be measured ... TBD.

(p) Weight percent solids and weight percent oxide methods are described in L\&H DQO Section 7.3.4. 
HNF-4577, Rev. 4

\subsection{SAMPLE CUSTODY}

The chain-of-custody form is initiated by the sampling team as described in the work packages. Samples are shipped in a cask and sealed with a Waste Tank Sample Seal (see below).

\begin{tabular}{||l|l||}
\hline \multicolumn{2}{|c||}{ WASTE TANK SAMPLE SEAL } \\
\hline Supervisor: & Sample No.: \\
\hline Date of Sampling: & Time of Sampling: \\
\hline Shipment No.: & Serial No.: \\
\hline
\end{tabular}

Each sample number shall be created using the sample's core and segment number. For instance, segment 1 of core 197 would be sample number 197-01. The sealed and labeled samples are shipped to the laboratory along with the chain-of-custody form. The receipt and control of samples in the 222-S Laboratory are described in laboratory procedure LO-090-101.

\subsection{EXCEPTIONS, CLARIFICATIONS, AND ASSUMPTIONS}

\subsection{EXCEPTIONS TO DAT A QUALITY OBJECTIVES REQUIREMENTS}

The solids dissolution rate determination required by the HLW and LAW DQOs is waived per customer request for tank 241-AZ-102 only. Consequently, no special testing steps are required when the solids and liquids are mixed during preparation of the composite.

The requirement to dry the solids fraction of the tank composite at $105^{\circ} \mathrm{C}$ ("solids B") prior to analysis (Patello et al. 1999) is waived per customer request for tank 241-AZ-102 only.

The L\&H DQO requires full dissolution of solids samples for analysis. However, some of the analyses in Table 2 can only be performed on water digests of solids; full dissolution of the sample for analysis is not currently possible for these analytes. These analytes are: TIC and TOC by furnace oxidation, anions by IC, tritium, carbon-14, and iodine- 129 .

Many of the analyses performed in triplicate as directed by the L\&H DQO are also required in duplicate by the HLW and LAW DQOs. Per customer request, duplication of effort is to be avoided by performing these analyses in triplicate as directed by the L\&H DQO to satisfy the requirements of the HLW and LAW DQOs in the event that dilution is not required. Tables 1 through 6 reflect compromises between these DQO requirements to satisfy the affected programs. Several analytes being measured by ICP/MS required by the L\&H DQO will take the place of ICP/AES analyses for liquids (Ba and $\mathrm{La}$ ) and solids (As, B, Be, Ce, Co, Cs, $\mathrm{Li}, \mathrm{Mo}, \mathrm{Pr}$, $\mathrm{Rb}, \mathrm{Sb}, \mathrm{Se}, \mathrm{Ta}, \mathrm{Te}, \mathrm{Th}, \mathrm{Tl}, \mathrm{V}$ ) required by the HLW and LAW DQOs. 


\section{HNF-4577, Rev. 4}

The tables identify many analytes to be determined by ICP/MS because this technique has the sensitivity to meet the desired MRQs. However if the concentration of the analyte is high enough the quality of the ICP/AES results will be as good as or better than the ICP/MS data. Because ICP/MS is based on the measurement of different element isotopes, the total amount of an element must be determined by summing the isotopes or by calculations assuming the natural abundance of the isotopes. However, in the production of nuclear materials these natural abundances are changed particularly around the mass peaks of the fission product yield curves (AMU 90 and 137). The presence of the fission products in the samples can lead to unnatural isobaric interferences which can lead to inaccuracies in the measurements. Another potential interference to the ICP/MS method is in the low ( $<80 \mathrm{AMU})$ atomic mass range where polyatomic species and ionized species from the argon plasma gas can cause interference problems. Elements in this region may be determined easier and more accurately using ICP/AES if the concentrations in the sample are high enough. Because it is not possible to precisely predict what trace analytes will be present in high enough concentrations for ICP/AES analysis, it is recommended that the ICP/AES be performed for all possible analytes. For liquid samples, the following elements cannot be analyzed by ICP/AES at the required MRQs: As, B, Ba, Be (borderline), $\mathrm{Ce}, \mathrm{Co}, \mathrm{K}, \mathrm{La}, \mathrm{Li}, \mathrm{Mo}, \mathrm{Sb}, \mathrm{Se}, \mathrm{Th}, \mathrm{Tl}$, and $\mathrm{V}$. For solid samples, the following elements cannot be analyzed by ICP/AES at the required MRQs: Ag (borderline), As, B, Ce, Co, $\mathrm{La}$ (borderline), $\mathrm{Li}, \mathrm{Mo}, \mathrm{Na}$ (borderline), Nd (borderline), $\mathrm{S}$ (borderline), $\mathrm{Sb}, \mathrm{Se}, \mathrm{Th}, \mathrm{Tl}, \mathrm{U}$ (borderline), V, Y, and $\mathrm{Zn}$ (borderline). The following analytes are expected to be semiquantitative for the ICP/MS method: $\mathrm{Ag}, \mathrm{Ba}, \mathrm{Mo}, \mathrm{Nd}, \mathrm{Pd}, \mathrm{Rb}, \mathrm{Ru}, \mathrm{Sb}, \mathrm{Se}, \mathrm{Te}$, and ${ }^{231} \mathrm{~Pa}$. Sb and $\mathrm{Se}$ can be performed by graphite furnace atomic absorption (GFAA) and are therefore requested by that method.

The 222-S laboratory does not have analytical methods for ${ }^{59} \mathrm{Ni},{ }^{63} \mathrm{Ni},{ }^{126} \mathrm{Sb},{ }^{126 \mathrm{~m}} \mathrm{Sb},{ }^{121 \mathrm{~m}} \mathrm{Sn},{ }^{93} \mathrm{Nb}$, or ${ }^{93} \mathrm{Zr}$.

For the Equipment DQO (Bloom 1996) only shear strength is required.

No analytical method is currently available to perform the sludge particle density measurements required by the HLW DQO. (Nguyen 1999a). These analyses may be requested at a later date if and when a method becomes available.

The HLW and H\&L DQOs require that full cores be obtained from two widely spaced risers. The sludge from riser $15 \mathrm{H}$ will be composited with supernate from another riser, $15 \mathrm{C}$. The supernate in tank $241-\mathrm{AZ}-102$ is expected to be laterally homogenous due to convective mixing driven by radioactive decay heat within the waste. Consequently, this composite will be representative of the tank contents at the riser $15 \mathrm{H}$ location.

The H\&L DQO requires that the combined liquids and solids slurry composite be subsampled and analyzed for weight percent oxides. This step is performed per a test plan (Person 1999), since analytical procedures are not in place.

\subsection{CLARIFICATIONS AND ASSUMPTIONS}

The laboratory is requested to report all analytical results recovered from multi-analyte methods, including the inductively coupled plasma - atomic emission spectroscopy (ICP/AES), gamma 


\section{HNF-4577, Rev. 4}

energy analysis (GEA), and ion chromatography (IC) analyses, even though only specific analytes are requested. These opportunistic analyses (Kristofzski 1996) should be reported only if no additional preparatory work is required (e.g., running additional standards) and if the error associated with the results is documented. No reruns or additional analyses should be performed to improve recovery for analytes not specifically requested in Tables 1 or 2 . 
HNF-4577, Rev. 4

\subsection{ORGANIZATION}

The organization and responsibility of key personnel involved with this tank 241-AZ-102 characterization project are listed in Table 7.

Table 7: Tank 241-AZ-102 Project Key Personnel List

\begin{tabular}{|c|c|c|}
\hline Responsibility & Organization & Individual \\
\hline $\begin{array}{l}\text { RPP 241-AZ-102 Tank } \\
\text { Coordinator }\end{array}$ & $\begin{array}{l}\text { RPP Process Engineering } \\
\text { (LMHC) }\end{array}$ & J. H. E. Rasmussen, 373-1128 \\
\hline $\begin{array}{l}\text { 222-S Laboratory Project } \\
\text { Coordinator }\end{array}$ & Analytical Services $(\mathrm{FH})$ & F. H. Steen, 372-2495 \\
\hline $\begin{array}{l}\text { 222-S Laboratory Point of } \\
\text { Contact (day shift) }\end{array}$ & Analytical Services (WMH) & W. I. Winters, 373-1951 \\
\hline $\begin{array}{l}\text { 222-S Laboratory Point of } \\
\text { Contact (off-hours) }\end{array}$ & Analytical Services (WMH) & $\begin{array}{l}\text { 222-S Laboratory Shift } \\
\text { Manager, 373-2435 }\end{array}$ \\
\hline $\begin{array}{l}200 \text { East Tank Farm Point of } \\
\text { Contact }\end{array}$ & Tank Farm Operations & $\begin{array}{l}\text { East Tank Farm Operations } \\
\text { Shift Manager, } 373-3475 \text { or } \\
373-2689\end{array}$ \\
\hline Data Management & $\begin{array}{l}\text { Manager, Data Development } \\
\text { and Interpretation }\end{array}$ & J. G. Field, 376-3753 \\
\hline $\begin{array}{l}\text { Process Engineering Point of } \\
\text { Contact for Immediate } \\
\text { Notifications }\end{array}$ & $\begin{array}{l}\text { RPP Process Engineering } \\
(\mathrm{LMHC})\end{array}$ & $\begin{array}{l}\text { On-Call Process Engineer } \\
539-2074 \text { or } 85-9654 \text { (pager) }\end{array}$ \\
\hline $\begin{array}{l}\text { Process Chemistry Point of } \\
\text { Contact }\end{array}$ & $\begin{array}{l}\text { Manager, Process Chemistry } \\
\text { (NHC) }\end{array}$ & L. L. Lockrem, 373-4471 \\
\hline $\begin{array}{l}\text { RPP Privatization Point of } \\
\text { Contact }\end{array}$ & Manager, Privatization Interface & K. A. Gasper, 373-1948 \\
\hline $\begin{array}{l}\text { Waste Feed Delivery Point of } \\
\text { Contact }\end{array}$ & $\begin{array}{l}\text { Waste Feed Delivery/Retrieval } \\
\text { Engineering }\end{array}$ & J. H. Baldwin, 373-4533 \\
\hline $\begin{array}{l}\text { Waste Process and } \\
\text { Development Point of } \\
\text { Contact }\end{array}$ & $\begin{array}{l}\text { Waste Process and Development } \\
\text { (PNNL) }\end{array}$ & I. E. Burgeson, $372-3650$ \\
\hline
\end{tabular}

\subsection{DELIVERABLES}

All analyses will be reported as Formats I, IV, or VI as indicated in Tables 1 and 2. Additional information regarding reporting formats is given in Thompson (1999). 


\section{HNF-4577, Rev. 4}

\subsection{FORMAT I REPORTIN G}

Tables 1 and 2 contain the notification limits for each analyte. Any results exceeding their notification limits shall be reported via telephone by the 222-S Laboratory Facility Planning Team to the East Tank Farm Operations shift manager as soon as the data are obtained and reviewed by the responsible scientist. This verbal notification must be followed within one hour by electronic notification to the tank farm operations shift manager, the River Protection Project (RPP) Process Engineering Data Development and Interpretation manager, the On-Call Process Engineer, and the tank coordinator responsible for the tank. Additional analyses for verification purposes may be contracted between the performing laboratory and the tank coordinator by either a revision to this SAP or by a letter.

\subsection{FORMAT IV REPORTING}

The format IV report shall be a data package reporting the results of analyses performed and will resemble a regulatory data package without third party validation. The data package should be prepared by tank and include the data for all samples, including (as applicable) formation of composites, segments, subsegments, drainable liquids, and associated blanks taken and analyzed for this sampling event and for core sample 254 which was obtained from tank 241-AZ-102 in 1998. The recommended reporting format and the raw data that shall be included are given in Section A5.0 of Schreiber (1998). The data package shall be issued 180 days after the last sample is received at the laboratory. The raw data shall be accessible to the program in accordance with the laboratory's Records Inventory and Disposition Schedule and until the respective waste tank is closed or the waste is treated.

In addition to this data package, an electronic version of the analytical results shall be provided to the Tank Characterization Database representative on the same day that the final data package is issued. The data must be available to the Washington State Department of Ecology within 7 days of release of the data package. The electronic version shall be in the standard electronic format (Lang et al., 1999).

\subsection{FORMAT VI REPORTING}

The Americium-241 analyses subsampled in triplicate and performed in duplicate for each solids segment per Poppiti (1999a) must be completed within 45 calendar days after the completion of the sampling event. The laboratory shall issue a letter report specific to this Americium-241 analysis within this 45-day period. The report shall include identification of the segment locations, the weight fraction of solids and liquids for each segment, and a LABCORE report including the Americium -241, total alpha, solids density, centrifuged solids density, and weight fraction of centrifuged solids measurements. The report shall be provided to Dr. Neil R. Brown, Waste Processing and Disposal Program, Mr. James F. Thompson, Jr. Program Development Division, Ms Karyn Wiemers, Chemical Process Development Group, and Ms Kathleen Hall, Data Development and Interpretation. 
HNF-4577, Rev. 4

\subsection{CHANGE CONTROL}

Under certain circumstances, it may become necessary for the performing laboratory to make decisions concerning a sample without review of the data by the customer or the Characterization Project. All significant changes (such as analyte additions or analysis of new, additional samples) shall be documented by RPP Process Engineering via an Engineering Change Notice to this SAP or by a letter. All changes shall also be clearly documented in the final data report. Insignificant changes may be made by the tank or project coordinator by placing a notation in the permanent record (i.e., note change in extrusion log book or memorandum to file). Significance is determined by the tank coordinator.

At the request of the Characterization Project, additional analysis of sample material from this characterization project shall be performed following a revision of this SAP or issuance of a letter. 


\section{HNF-4577, Rev. 4}

\subsection{REFERENCES}

Bauer, R. E., and L. P. Jackson, 1998, Data Quality Objective to Support Resolution of the Flammable Gas Safety Issue, HNF-SD-WM-DQO-004, Rev. 3-A, Lockheed Martin Hanford Corporation, Richland, Washington.

Bloom, G. R., 1996, Characterization Data Needs for Development, Design, and Operation of Retrieval Equipment Developed through the Data Quality Objective Process., WHC-SDWM-DQO-008, Rev. 1, Westinghouse Hanford Company, Richland, Washington.

Board, D.C., 1998, Tank Waste Remediation System, Characterization Project, Quality Policies, HNF-SD-WM-QAPP-025, Rev. 4, Lockheed Martin Hanford Corporation, Richland, Washington.

Brown, T. M, J. W. Hunt, and L. J. Fergestrom, 1998, Tank Characterization Technical Sampling Basis, HNF-SD-WM-TA-164, Rev. 4, Lockheed Martin Hanford Corporation, Richland, Washington.

CHG, 1999, Nonconcforming Item Reporting and Control, RPP-PRO-298, Rev. 0, CH2M Hill Hanford Group, Inc., Richland, Washington.

DOE-RL, 1998, Hanford Analytical Services Quality Assurance Requirements Document, DOE/RL-96-68, Rev. 2, U.S. Department of Energy, Richland Operations Office, Richland, Washington.

Dukelow, G. T., J. W. Hunt, H. Babad, and J. E. Meacham, 1995, Tank Safety Screening Data Quality Objective, WHC-SD-WM-SP-004, Rev. 2, Westinghouse Hanford Company, Richland, Washington.

Esch, R. A. 1997a, Tank Waste Remediation System (TWRS) Privatization Contractor Samples for Low Activity Waste Envelope A, Tank 241-AW-101, Final Analytical Report, HNF-SD-WM-DP-204, Rev. 1, Rust Federal Services of Hanford, Inc., Richland, Washington.

Esch, R. A. 997b, Tank Waste Remediation System (TWRS) Privatization Contractor Samples for Low Activity Waste Envelope A, Tank 241-AN-105, Final Analytical Report, HNF-SD-WM-DP-218, Rev. 1, Rust Federal Services of Hanford, Inc., Richland, Washington.

Esch, R. A. 1997c, Tank Waste Remediation System (TWRS) Privatization Contractor Samples for Low Activity Waste Envelope A, Tank 241-AN-107, Final Analytical Report, HNF-SD-WM-DP-205, Rev. 1, Rust Federal Services of Hanford, Inc., Richland, Washington.

Hanlon, B M., 1999, Waste Tank Summary Report for Month Ending February 28, 1999, HNF-EP-0182-131, Lockheed Martin Hanford Corporation, Richland, Washington. 


\section{HNF-4577, Rev. 4}

Herting, D. L. 1999, Test Plan for Retrieval Testing of Sludge from Tanks 241-AZ-101, 241-AZ102, and 241-AY-102, HNF-5491, Fluor Daniel Hanford, Inc., Richland, Washington.

Jewett, J. R., 1999, Letter of Instruction for 241-AZ-102 Process Test (Interoffice Memorandum 82400-99-056 to K. M. Hall, October 13), Lockheed Martin Hanford Corporation, Richland, Washington.

Kristofzski, J. G., 1996, Directions for "Opportunistic Analyses," (Interoffice memorandum 75310-96-168, to J. H. Baldwin et al., September 11), Westinghouse Hanford Company, Richland, Washington.

Lang, L. L., S. F. Bobrowski, and S. J. Harris, 1999, Standard Electronic Format Specification for Tank Characterization Database Loader, Version 2.4, HNF-3638, Rev. 0, prepared by Pacific Northwest National Laboratory for Lockheed Martin Hanford Corporation, Richland, Washington.

Markel, L. P., 1999, 222-S Laboratory Quality Assurance Plan, HNF-SD-CP-QAPP-016, Rev. $3 \mathrm{C}$, Waste Management Hanford, Inc., Richland, Washington.

Nguyen, D. M., 1999a, Data Quality Objectives for TWRS Privatization Phase 1: Confirm Tank $T$ is an Appropriate Feed Source for High-Level Waste Feed Batch X, HNF-1558, Rev 1, Lockheed Martin Hanford Corporation, Richland, Washington.

Nguyen, D. M., 1999b, Data Quality Objectives for TWRS Privatization Phase 1: Confirm Tank $T$ is an Appropriate Feed Source for Low-Activity Waste Feed Batch X, HNF-1796, Rev 2, Lockheed Martin Hanford Corporation, Richland, Washington.

Patello, G. K., M. J. Truex, and K. D. Wiemers, 1999, Low-Activity Waste and High Level Waste Feed Data Quality Objectives, PNNL-12163, Pacific Northwest National Laboratory, Richland, Washington.

Person, J. C., 1999, Test Plan for Tank 241-AZ-102 Solubility Screening Tests, HNF-4751, Rev. 0 , Numatec Hanford Corporation, Richland, Washington.

Poppiti, J. A., 1999a, Contract No. DE-AC-06-96RL13200 - Additional Analysis of Double-Shell Tank 241-AZ-101 and 241-AZ-102 for Americium-241, (memorandum 99-PDD-047 to R. D. Hanson, June 10, 1999), United States Department of Energy Office of River Protection, Richland, Washington.

Poppiti, J. A., 1999b, Contract No. DE-AC-06-96RL13200 - Additional Tank 241-AZ-102 Sample Material to Support Privatization and Retrieval, (memorandum 99-PDD-068 to R. D. Hanson, September 9, 1999), United States Department of Energy Office of River Protection, Richland, Washington.

Poppiti, J. A., 1999c, Authorization to Modify and Delay Americium-241 Analysis on DoubleShell Tank 241-AZ-102, (memorandum 99-PDD-087/9958129 to M. P. Delozier, October 27, 1999), United States Department of Energy Office of River Protection, Richland, Washington. 


\section{HNF-4577, Rev. 4}

Reynolds, D. A., W. L. Cowley, J. A. Lechelt, B. C. Simpson, and C. Defigh-Price, 1999, Evaluation of Tank Data for Safety Screening, HNF-4217, Rev. 0, Lockheed Martin Hanford Corporation, Richland, Washington.

Schreiber, R. D., 1995, Tank Characterization Report for Double-Shell Tank 241-AZ-102, HNF-SD-WM-ER-411, Rev.0A, Lockheed Martin Hanford Corporation, Richland, Washington.

Thompson, R. R., 2000, Letter of Intent for River Protection Project (RPP) Characterization Program: Process Engineering, Hanford Analytical Services, Characterization Project Operations, and Quality Assurance, RPP-5539, CH2M Hill Hanford Group, Inc., Rev. 0, Richland, Washington. 
HNF-4577, Rev. 4

APPENDIX A:

EXPECTED PHYSICAL PROFILE OF TANK 241-AZ-102 CORE SAMPLES 
HNF-4577, Rev. 4

\section{APPENDIX A:}

\section{EXPECTED PHYSICAL PROFILE OF TANK 241AZ-102 CORE SAMPLES}

\section{Table A-1: Tank 241-AZ-102 Physical Profile Estimate Risers $15 C, 15 H$, and $24 A$}

\begin{tabular}{|c|c|c|l|l|}
\hline Segment \# & Inches & $\begin{array}{c}\text { Elevation Range } \\
\text { (ft. MSL) }\end{array}$ & \multicolumn{1}{|c|}{ Waste Type } & \multicolumn{1}{|c|}{ Comments } \\
\hline 1 & 12.0 & $645.29-644.29$ & Air & \\
\cline { 2 - 6 } & 7.0 & $644.29-643.71$ & Liquid & Good Recovery \\
\hline 2 & 19.0 & $643.71-642.12$ & Liquid & Good Recovery \\
\hline 3 & 19.0 & $642.12-640.54$ & Liquid & Good Recovery \\
\hline 4 & 19.0 & $640.54-638.96$ & Liquid & Good Recovery \\
\hline 5 & 19.0 & $638.96-637.37$ & Liquid & Good Recovery \\
\hline 6 & 19.0 & $637.37-635.79$ & Liquid & Good Recovery \\
\hline 7 & 19.0 & $635.79-634.21$ & Liquid & Good Recovery \\
\hline 8 & 19.0 & $634.21-632.62$ & Liquid & Good Recovery \\
\hline 9 & 19.0 & $632.62-631.04$ & Liquid & Good Recovery \\
\hline 10 & 19.0 & $631.04-629.46$ & Liquid & Good Recovery \\
\hline 11 & 19.0 & $629.46-627.87$ & Liquid & Good Recovery \\
\hline 12 & 19.0 & $627.87-626.29$ & Liquid & Good Recovery \\
\hline 13 & 19.0 & $626.29-624.71$ & Liquid & Good Recovery \\
\hline 14 & 19.0 & $624.71-623.12$ & Liquid & Good Recovery \\
\hline 15 & 19.0 & $623.12-621.54$ & Liquid & Good Recovery \\
\hline 16 & 19.0 & $621.54-619.96$ & Liquid & Good Recovery \\
\hline 17 & 3.2 & $619.96-619.69$ & Liquid & Good Recovery \\
\cline { 2 - 5 } & 15.8 & $619.69-618.37$ & $\begin{array}{l}\text { Soft sludge, } \\
\text { NCAW waste. }\end{array}$ & Good Recovery \\
\hline 18 & 19.0 & $618.37-616.79$ & $\begin{array}{l}\text { Soft sludge, } \\
\text { NCAW waste. }\end{array}$ & Good Recovery \\
\hline
\end{tabular}

Note: elevations based on inside tank bottom elevation for Tank 241-AZ-102 of $616.54 \mathrm{ft}$. MSL MSL $=$ Mean Sea Level

NCAW $=$ Neutralized Current Acid Waste 
HNF-4577, Rev. 4

Figure A-2: Physical/Chemical Profile Estimate Chartof Tank 241-AZ-102

$R$ is e r $15 \mathrm{C}$

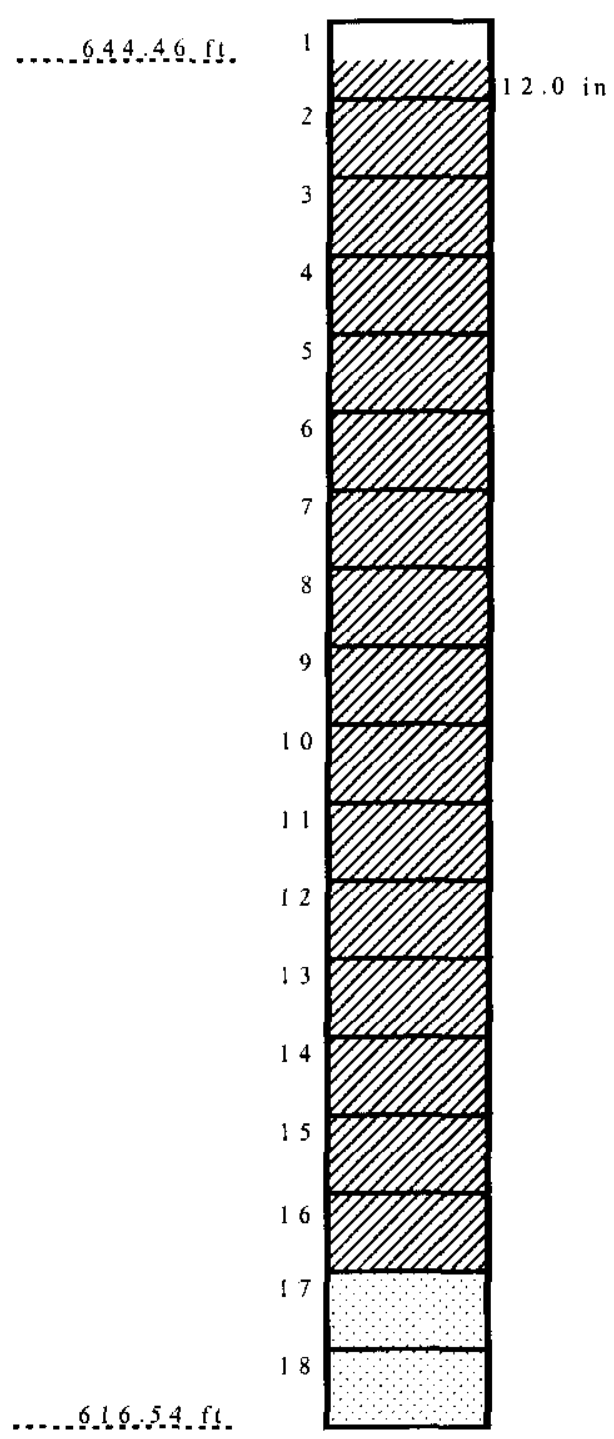

$R$ is e r $15 \mathrm{H}$

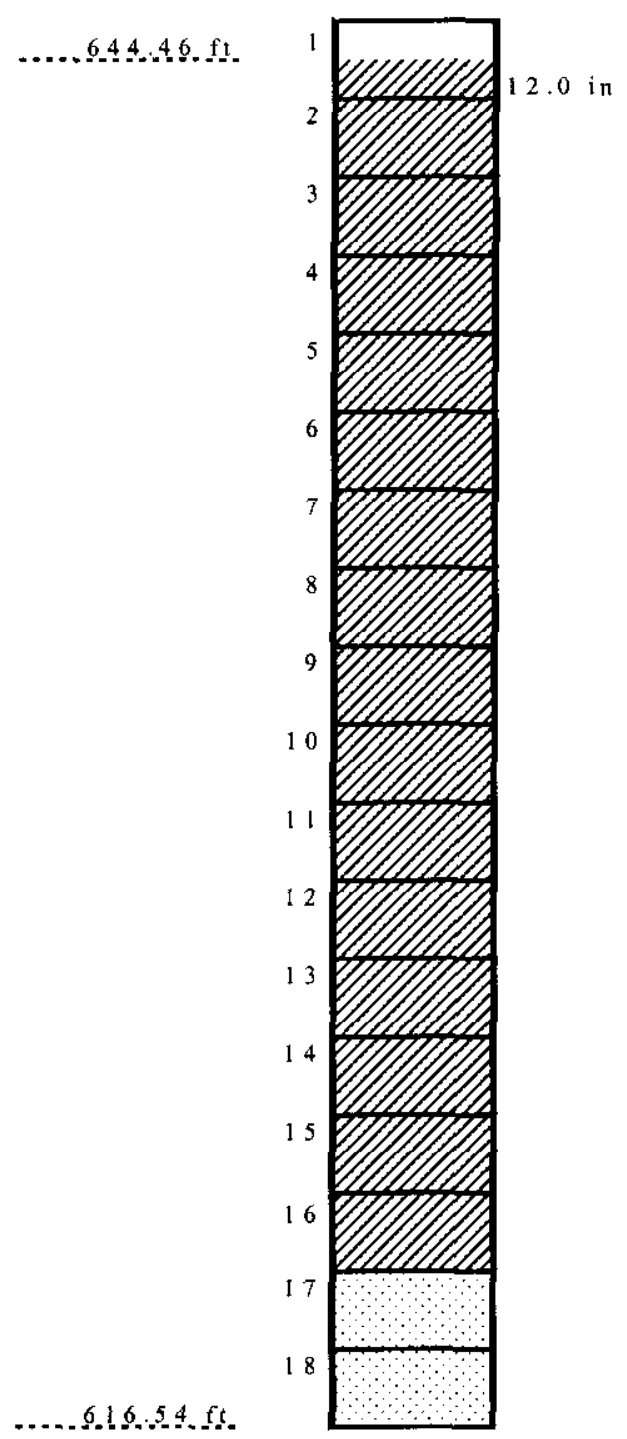

$R$ is er $24 \mathrm{~A}$

-...64. $64.46 .5 \mathrm{ft}$

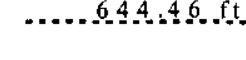

.....6. $6.6 .54 . \mathrm{ft}$

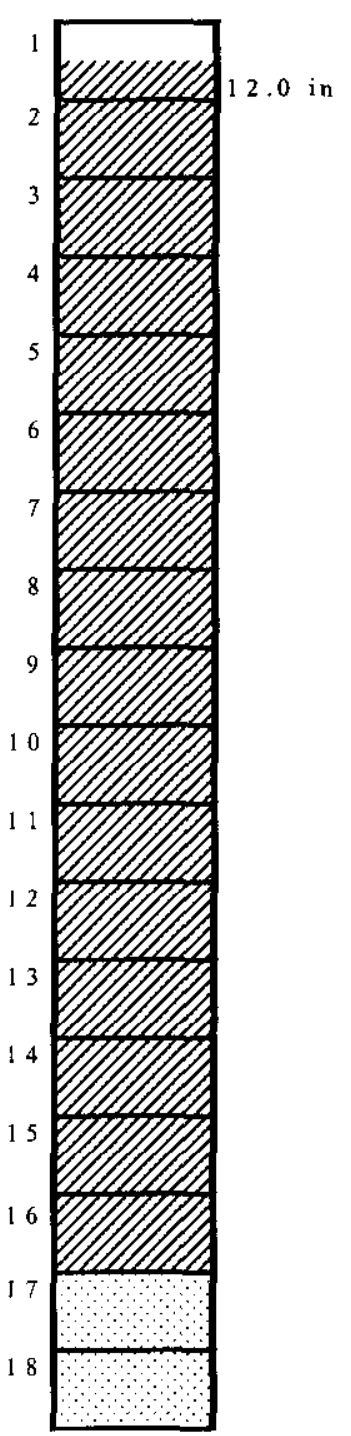

Note:

1. The waste level is based on a tank inside bottom elevation of $616.54 \mathrm{ft} \mathrm{M} \mathrm{SL}$ 2. Each segment is 19 inch long and partial segments werespecified

3. Wastedepth based on the manual tape measurement of $6 / 21 / 99$

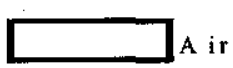




\section{HNF-4577, Rev. 4}

\section{APPENDIX B:}

\section{1-AZ-102 WASTE COMPOSITE PREPARATION GUIDELINES}

These preparation steps for the 241-AZ-102 waste composite satisfies the HLW, LAW and the L\&H DQO objective that the composite be representative of the waste to be retrieved and allows a master composite to be prepared for each riser in a single jar. Each riser composite is homogenized and subsampled for analysis and testing.

\section{Core Sample Extrusion:}

Cores shall be extruded onto the extrusion tray. Solids and drainable liquids shall be placed in separate jars. A chemist shall note the degree of separation achieved both for the solids and the drainable liquids. If the drainable liquid contains a high concentration of solids, the chemist needs to determine if centrifuging is needed to separate the solids. The composite preparation assumes minimal solids in the drainable liquids and minimal liquid in the extruded solids. Error is introduced in the composite preparation when there is a high concentration of solids in the drainable liquid because of the difficulty in keeping the solids suspended during the liquid transfer into the composite jars. When excess liquid is in the solids, again maintaining homogeneity of the transferred solids is difficult. When making a decision to centrifuge, the composite preparation errors need to be balanced with errors associated with increased sample handling and transfer associated with the centrifuging operation.

During the entire sample extrusion process and possible subsequent sample preparation, a thorough material balance needs to be maintained and unaccounted for mass loss shall be less than $10 \%$. Observations of sample jars are very important to understanding and interpretation of later analyses.

\section{Composite Preparation:}

The composite preparation steps assume that each core segment has a jar of drainable liquids and a jar of solids. If a segment has only one jar, the steps below still apply. The number of composite jars prepared is dependent on the quantity of composite needed to meet the requirements in the Sampling and Analysis Plan (SAP).

Use the following steps to prepare a set of whole tank composites in individual composite jars.

1. Based on the quantity of composite required, the number of segments, and distribution of segments sampling locations in the tank, determine the weight of solids required from each segment to prepare each individual composite jar (reference calculations in the L\&H DQO).

2. 


\section{HNF-4577, Rev. 4}

3. Based on the quantity of composite required, the number of segments, and distribution of segments sampling locations in the tank, determine the weight of liquids required from each segment to prepare each of the composite jars (reference calculations in the DQO).

4. Homogenize the contents of the first segment solids jar by mixing.

5. Transfer a solids aliquot from the homogenized segment solids jar (weight calculated above) into the solids composite jar.

6. Repeat steps 4 through 5 for each segment to be used in preparing the solids composites. Note that homogenization of the sample jar before transferring an aliquot is important.

Preparation of the solids composites is now complete. The analyses and tests per the LAW, HLW, and L\&H DQOs may entail differing ratios of liquids to solids. Preparation of the combined liquids and solids composite for analysis per Tables 1 and 2 is described below. Those composites to be used for testing per the LAW, HLW, and L\&H DQOs will be prepared according to the respective test plans prepared for those tests.

7. Subsample the each riser solids composite in order to provide sufficient solids for analyses in triplicate and re-analysis per Table 2 . Note that for Tank 241-AZ-102 the quantity of solids is limiting; sufficient liquids will be available for analysis per Table 1 if sufficient solids are included in the composite for analysis per Table 2 .

8. Homogenize the contents of the first segment liquids jar by mixing.

9. Distribute a liquid aliquot from the homogenized segment liquid jar (weight calculated above) into each individual composite jar.

10. Repeat steps 8 through 9 for each segment to be used in preparing the composites. Note that homogenization of the sample jar before transferring an aliquot is important.

The composite preparation is now complete. The following measurements are required for the composites.

11. Allow the composite jars to settle undisturbed for 12 to 24 hours.

12. Measure and document the volume \% settled solids for each composite jar.

Compositing steps shall be conducted in the presence of a chemist. The attending chemist is to compare jar-to-jar variability based on observed volume \% settled solids. Any adjustments to the composite are to be documented and are to accommodate preservation of the sample pedigree to the extent possible. The attending chemist shall direct adjustments to the composite. 


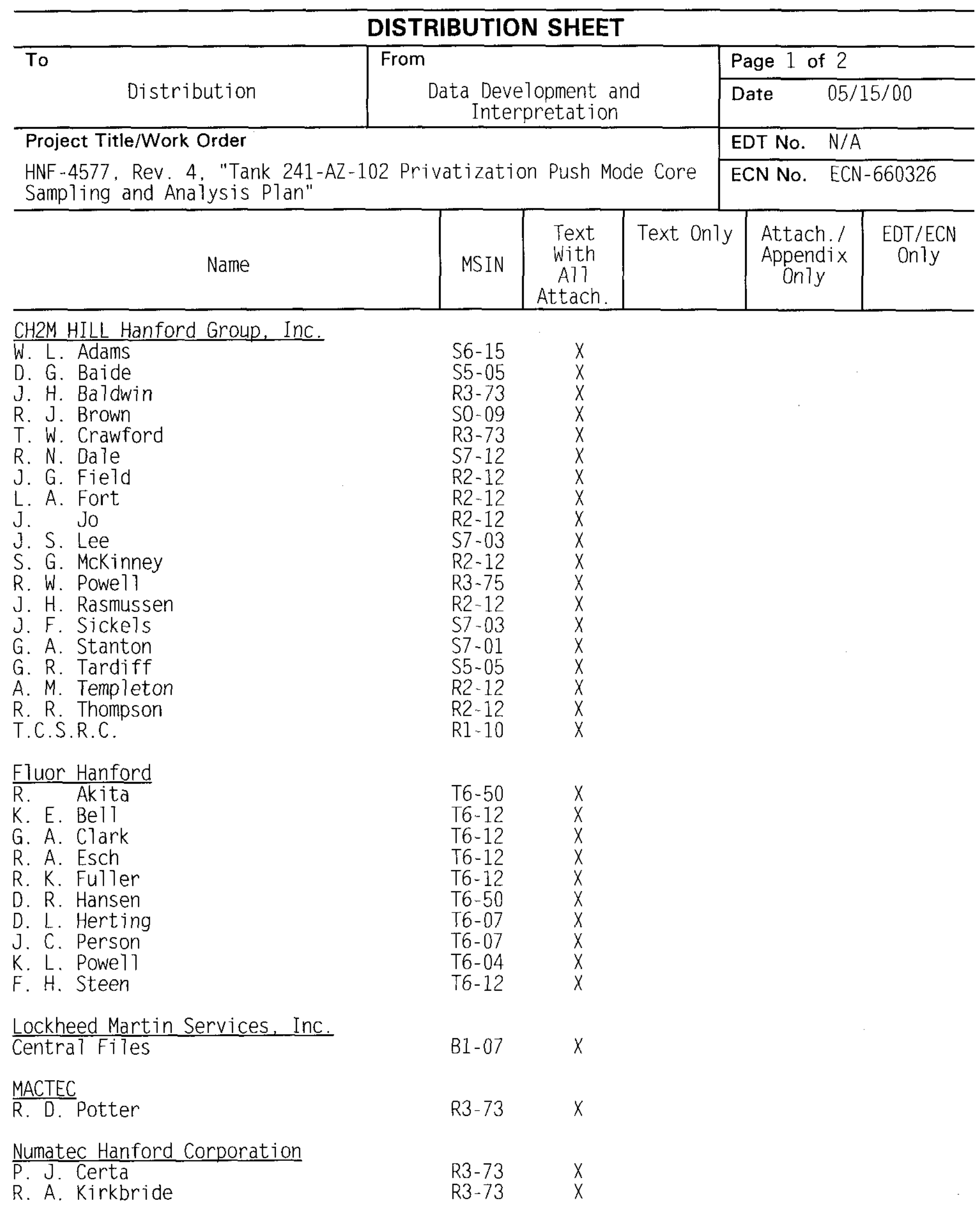




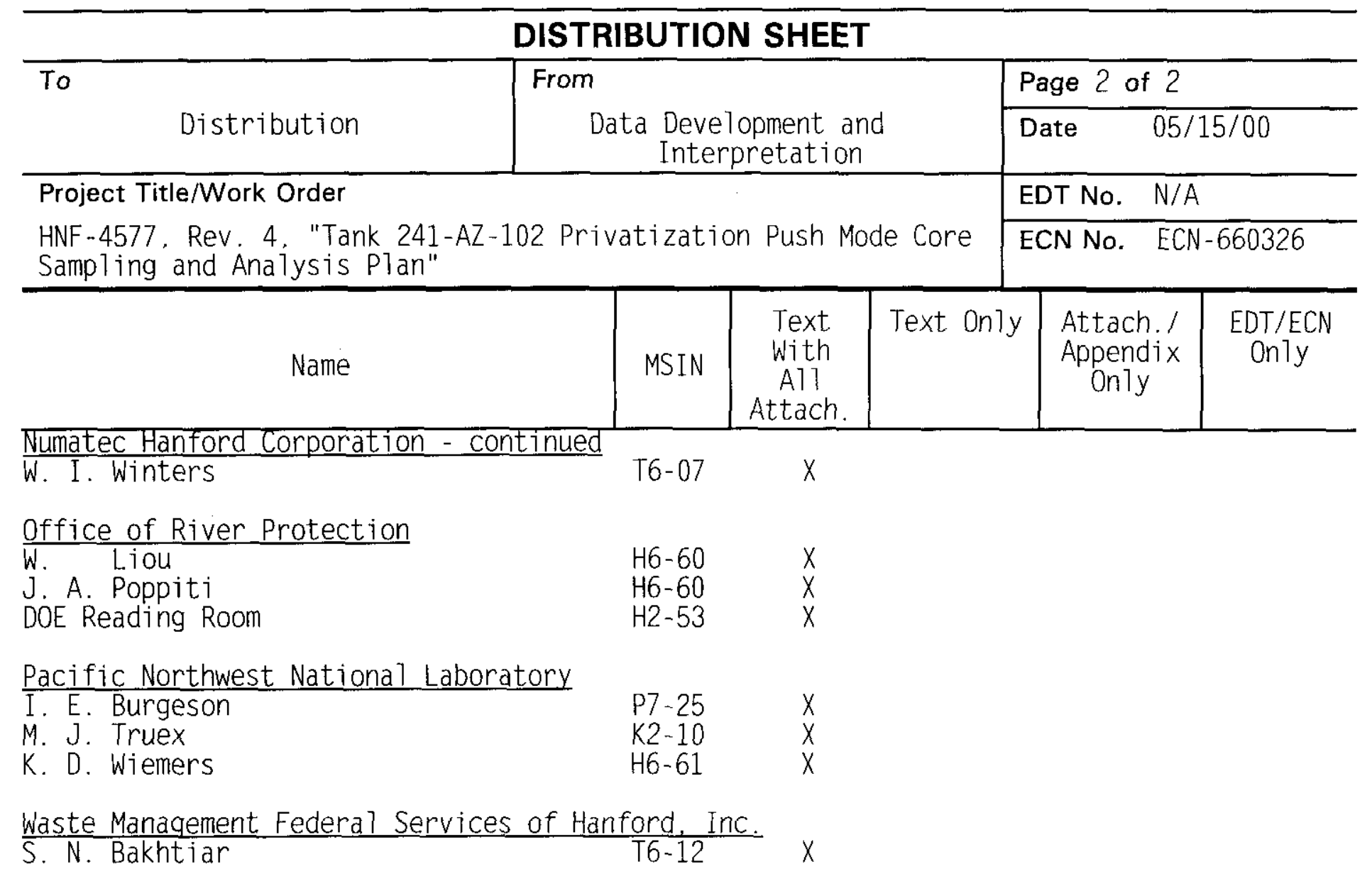

